Ann. Sci. forest., 1968, 25 (4), 251-289.

\title{
INFLUENCE DES CONDITIONS DE NUTRITION MINÉRALE SUR LE DÉPÉRISSEMENT DU PIN MARITIME DANS LES LANDES DE GASCOGNE
}

\author{
M. BONNEAU, J. GELPE, F. LE TACON \\ avec la collaboration technique de A. Clement, B. Lefrou, M. Bitsch, \\ A. RANC, D. SALMON \\ Station de Recherches forestières de Rordeaux, 33 - Cestas \\ Station de Recherches sur les Sols forestiers et la Fertilisation, \\ Centre national de Recherches forestières, 54- Nancy \\ Institut national de la Recherche agronomique
}

SOMMAIRE

Le dépérissement du Pin maritime d'origine portugaise dans les landes de Gascogne peut être attribué à la conjonction de l'origine des graines et de graves insuffisances de nutrition. Une fertilisation à base d'azote et de phosphore permet le rétablissement d'une vigueur normale et augmente considérablement la production ligneuse.

\section{1. - INTRODUCTION}

A partir de 1963, les sylviculteurs landais ont vu avec inquiétude de nombreux reboisements de Pin maritime de 5 à 15 ans environ, effectués à la suite des grands incendies de 1947 et 1949 , donner des signes de dépérissement accusés : chute précoce des aiguilles qui ne subsistaient que 1 ou 2 ans au lieu de 3 ans normalement, donnant ainsi aux arbres un aspect dénudé très anormal, apparition de chancres dans la partie supérieure de la tige et finalement mortalité importante.

Les services de la Direction des Forêts estimaient en 1965 que 100000 ha de reboisements étaient ainsi gravement compromis (soit $1 / 10$ du massif landais) ; ils étaient localisés surtout dans les zones les plus humides et notamment dans la Grande Lande. Les peuplements issus de graines récoltées par les propriétaires eux- 
mêmes sur des arbres de la région n'étaient presque jamais atteints par ces dépérissements qui se manifestaient dans la quasi-totalité des cas sur des semis issus de graines achetées dans le commerce et provenant en grande majorité du Portugal.

Trois causes possibles pouvaient être avancées pour expliquer ces dépérissements :

a) une inadaptation des Pins portugais au climat local : ils auraient en particulier souffert du rude hiver 1962-1963 (Bouvarel) ;

b) une attaque cryptogamique : en effet les chancres étaient presque toujours couverts des fructifications d'un champignon, le calliciopsis pinea ;

c) une nutrition minérale insuffisante, hypothèse logique à formuler après les résultats spectaculaires des essais de fertilisation entrepris par divers organismes sur les semis de Pin d'origine landaise.

C'est la dernière de ces hypothèses que nous nous sommes proposés de vérifier.

\section{2. - DÉPÉRISSEMENT ET TYPE DE SOL}

Nous nous sommes tout d'abord attachés à déterminer s'il pouvait exister une liaison entre la présence, l'absence ou l'intensité du dépérissement et le type de sol.

Il était donc indispensable de chiffrer ou d'estimer l'intensité du dépérissement. En 1965, nous avons adopté une méthode d'estimation à l'œil qui avait l'avantage d'être rapide mais dont le principal défaut était de manquer d'objectivité.

Nous avons utilisé l'échelle suivante :

- 0 peuplement ayant presque disparu

$\left.\begin{array}{l}\text { - I. } \\ \text { - II. }\end{array}\right\}$ peuplement très atteint

- III. peuplement moyennement atteint

- IV. peuplement peu atteint

$\left.\begin{array}{l}\text { - V. } \\ \text { - VI. }\end{array}\right\}$ peuplement sain

Cette notation adoptée, nous avons essayé de classer tous les sols que nous avons rencontrés dans la zone atteinte par le dépérissement.

\section{1. - Les sols landais}

Nous pouvons distinguer trois grandes catégories de sols :

- les sols squelettiques du littoral :

- les sols de la zone périphérique :

— les sols du plateau landais ; 
Nous passerons ici, sous silence, les sols des dunes car il n'y existe pas de peuplements de Pins maritimes d'origine portugaise. D'autre part, nous ne décrirons cidessous que les types de sols les plus largement répandus et qui englobent $95 \%$ des profils que nous avons pu observer.

\subsection{Les sols de la zone périphérique}

Le Pin maritime ne s'y trouve guère que sur les “ sables fauves " comme ceux de Chalosse qui sont plus riches en éléments fins que ceux des Landes proprement dites. La teneur en argile est comprise entre 10 et $15 \%$.

Ces sables parfaitement drainés ne présentent souvent aucune trace d'hydromorphie et, si intrinsèquement ils sont pauvres chimiquement, leur teneur en éléments assimilables est néanmoins beaucoup plus élevée que celle de la plupart des autres sols landais.

Génétiquement, les sols développés sur ces sables fauves de bordure sont des sols bruns acides et au point de vue forestier ils sont certainement les plus favorables que l'on puisse rencontrer dans les Landes.

\subsection{Les sols du plateau landais}

Ils se sont développés sur un matériel sableux grossier ne contenant jamais plus de $5 \%$ d'éléments fins (argile et limons).

Le facteur principal de différenciation des sols est l'existence à profondeur variable d'une nappe permanente, qui conditionne l'intensité et la profondeur de la migration des colloïdes organiques et des sesquioxydes libres.

Tous ces sols en effet appartiennent au groupe des podzols, sauf lorsque l'hydromorphie devient trop importante, empêchant ainsi toute migration.

Lors du travail de terrain, nous avons distingué six types de sols (fig. 1), où il est possible de ranger $95 \%$ des sols sur lesquels le dépérissement du Pin maritime sévit.

Ces six types sont classés d'après l'intensité de l'hydromorphie.

\section{Type $\mathrm{n}^{\circ} 1$ - Podzol non hydromorphe}

Ce type est caractérisé par une migration importante : I'horizon $\mathrm{A}_{2}$ cendreux est très épais (généralement plus de $40 \mathrm{~cm}$ et parfois davantage) et contient moins de $1 \%$ de matière organique.

L'horizon d'accumulation contient plus de $3 \%$ de matière organique ayant provoqué la consolidation des particules sableuses et la formation d'un alios.

Type $\mathrm{n}^{\circ} 2$ - Podzol légèrement hydromorphe à $A_{2}$ différencié et à alios.

L'horizon $\mathrm{A}_{2}$ bien que très visible est peu épais (moins de $40 \mathrm{~cm}$ ). Il contient $1,5 \%$ de matière organique. 


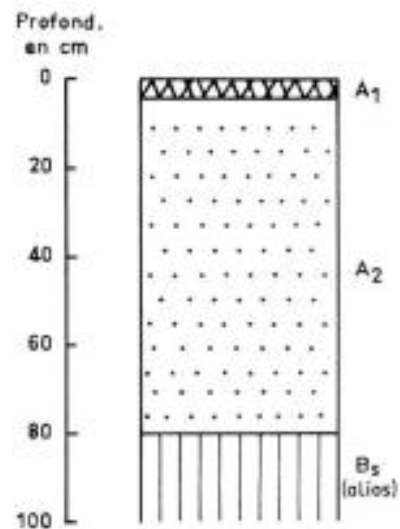

Type $n^{*} 1$

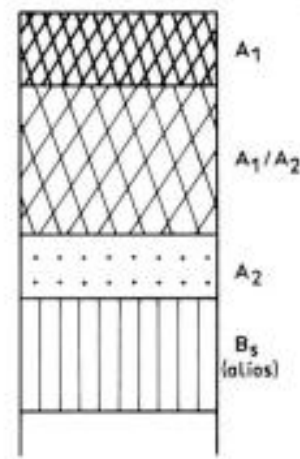

Type $n^{\circ 2}$

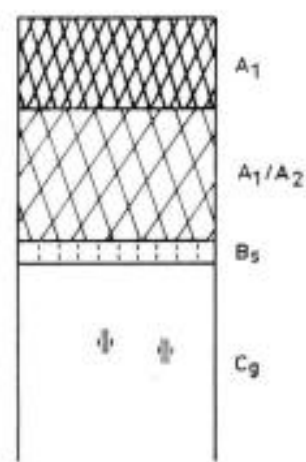

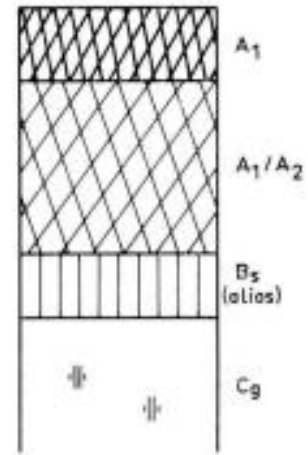

Type $\mathrm{n}^{\circ} 3$

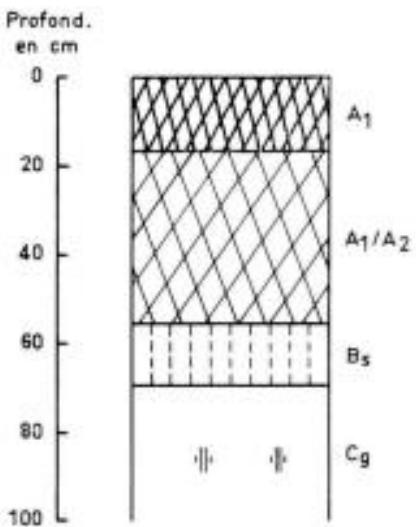

Type $\mathrm{n}^{\circ} 4$
Type $n^{0} 5$

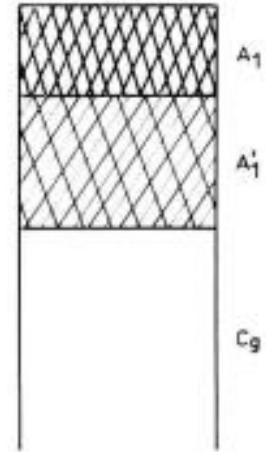

Type $n^{*} 6$

FIG. 1. - Les sols du plateau landais : aspect morphologique.

L'horizon d'accumulation est encore un alios contenant plus de $3 \%$ de matière organique mais il est moins épais que dans le cas du type 1.

La différenciation de ce sol a été influencée par l'existence d'une nappe permanente mais qui est suffisamment profonde pour ne pas entraver la formation d'un véritable horizon cendreux, ni celle d'un alios.

\section{Type $\mathrm{n}^{\circ} 3$ - Podzol hydromorphe à alios et sans horizon $A_{2}$ différencié}

L'hydromorphie a empêché la formation d'un horizon $\mathrm{A}_{2}$ véritable. La teneur en matière organique ne passe pas par un minimum comme pour les sols précédents. 
Nous pouvons seulement distinguer un horizon $A_{1} / A_{2}$ contenant un peu plus de $3 \%$ de matière organique, moins riche que l'horizon $A_{1}$.

L'hydromorphie n'a cependant pas entravé la formation d'un horizon B contenant encore $3 \%$ de matière organique et qui est encore un véritable alios, ni l'entrainement de l'aluminium.

\section{Type $\mathrm{n}^{\circ} 4-$ Podzol hydromorphe sans alios}

II n'existe qu'un horizon $A_{1} / A_{2}$ ayant une teneur en matière organique de l'ordre de $3,5 \%$.

Cette fois la nappe a fortement limité la migration des collö̈des humiques. Il existe bien un horizon B spodique assez épais $(20-30 \mathrm{~cm})$, mais qui ne contient plus que $1 \%$ environ de matière organique, ce qui est insuffisant pour cimenter les grains de sable entre eux et donc pour provoquer la formation d'un alios.

On constate d'autre part un renversement de la dynamique de l'aluminium à ce stade. La teneur en aluminium devient constante dans les trois horizons $A_{1}, A_{1} / A_{2}$ et B.

Il existe un horizon $C_{g}$, qui n'est pas un véritable horizon de gley. Cet horizon, même au niveau de la nappe en été, est jaune clair, sans trace de phénomènes de réduction permanente. Tout au plus observe-t-on parfois quelques taches rouille traduisant une réduction temporaire avec ensuite redistribution et réoxydation du fer.

\section{Type $\mathrm{n}^{\circ} 5$ - Podzol hydromorphe à horizon spodique réduit}

En raison de la proximité de la nappe qui peut même submerger le sol une partie de l'année, les processus de migration sont réduits au minimum. Seule une faible quantité de matière organique a pu migrer, constituant un horizon spodique de très faible épaisseur $(5-10 \mathrm{~cm})$.

L'horizon $\mathrm{C}_{\mathrm{g}}$ est de même nature que celui du sol de type 4 .

\section{Type $\mathrm{n}^{\circ} 6-$ Sol humique à gley}

Comme pour les sols hydromorphes précédents et pour les mêmes raisons, malgré la présence d'une nappe permanente à faible profondeur, il n'existe pas de véritable horizon de gley. Parfois cependant, on observe sous l'horizon $A_{1}$, un horizon entièrement blanchi d'où le fer a entièrement disparu. Mais dans la plupart des cas, ce que nous appelons $\mathrm{C}_{\mathrm{g}}$ ne se distingue en rien du sable originel.

Le rapport $\mathrm{C} / \mathrm{N}$ est relativement bas, de l'ordre de 20 , alors qu'il est beaucoup plus élevé, de l'ordre de 35 à 40 , dans les podzols non hydromorphes de type 1 . Ce phénomène est en relation avec la prédominance d'Éricacées sur les sols drainés, alors que, sur les sols hydromorphes, la molinie forme l'essentiel de la couverture herbacée.

Enfin la teneur en aluminium libre présente un maximum dans les horizons supérieurs. 
_- Matiàre orgenique

.... Aluminium tibre

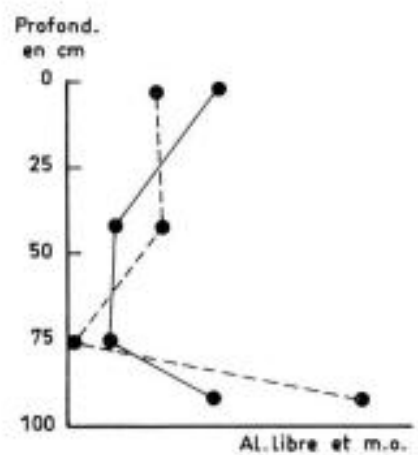

Type $\mathrm{n}^{\circ} 1$

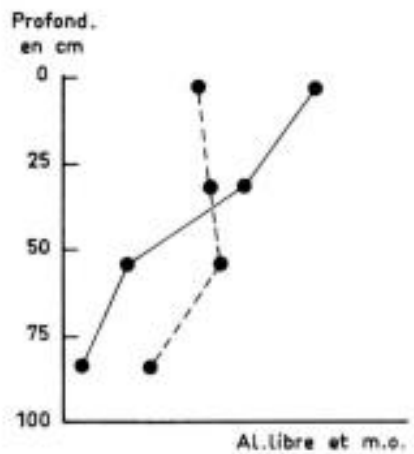

Type $n^{*} 4$

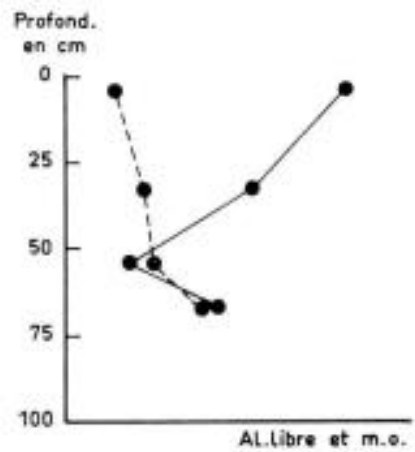

Type $\mathrm{n}^{\circ} 2$

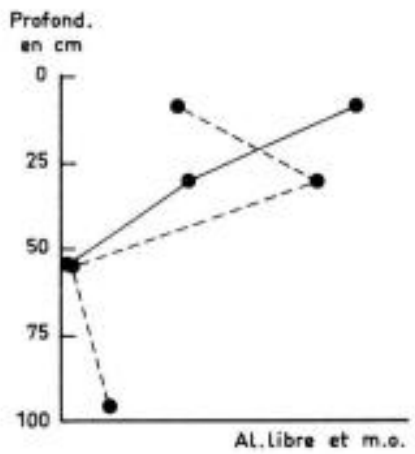

Type $n^{*} 6$

FIG. 2. - Les sols du plateau landais : variation de la teneur en aluminium libre et en matière organique en fonction de la profondeur

\section{2. - Variation de l'intensité du dépérissement en fonction du type de sol}

\subsection{Propriété Rasson}

Nous avons tout d'abord essayé de résoudre le problème dans un cas particulier. Nous avons choisi un reboisement de 150 hectares (propriété Rasson - commune de Retjons), effectué après incendie en 1952, en raison des grandes variations que l'on pouvait observer dans l'intensité du dépérissement.

Cette propriété avait d'autre part l'avantage de présenter côte à côte des pins des deux origines : landaise et portugaise. Une partie du contrat s'est en effet régénérée 
naturellement en pins du pays après l'incendie et le reste a été semé artificiellement avec des graines achetées dans le commerce et vraisemblablement portugaises.

Grâce à une photographie aérienne, prise juste après la plantation, il nous a été très facile de délimiter les deux origines sur le terrain, ce qu'il est très difficile sinon impossible de faire normalement, uniquement d'après les caractéristiques morphologiques des arbres.

Deux cartes, celle du sol et celle du peuplement, non publiées ici, ont été levées à grande échelle (1/5000). Le levé de la carte des sols a été effectué par sondages systématiques et utilisation de la photographie aérienne, à l'aide de laquelle il était possible de distinguer les zones hydromorphes et les zones drainées.

Nous avons ainsi délimité les six types de sols précédemment décrits.

La carte du peuplement plus difficilement réalisable a été effectuée en utilisant le système de notation du dépérissement (échelle de 0 à VI).

Par comparaison des deux cartes, il a été rapidement possible de dégager les premières conclusions.

\subsection{Comportement du Pin landais}

Les Pins landais se sont installés dans la partie sud-ouest du contrat, sur deux types de sols opposés. Ils se sont en effet régénérés sur la totalité d'une ancienne dune caractérisée par un podzol à $\mathrm{A}_{2}$ différencié du type 1 et sur une partie d'une dépression très mal drainée avec des sols de type 5 et 6 .

Dans ces deux stations très différentes les peuplements sont très convenables et ne manifestent aucun signe de dépérissement, même si leur croissance n'est pas tout à fait identique.

\subsection{Comportement du Pin portugais}

Le Pin portugais a été semé sur tous les types de sols sauf sur le type $n^{\circ} 1$ entièrement occupé par le pin de race locale. La superposition des deux cartes permet de constater que le dépérissement ne se manifeste pas au hasard. Le dépérissement est maximum sur les sols de type 5 et 6 , donc sur les sols les plus hydromorphes, et minimum sur les sols de types 2 et 3 beaucoup moins influencés par la nappe d'eau.

L'utilisation du test du $\chi^{2}$ de Karl PEARSON permet de mettre en évidence statistiquement cette liaison avec une probabilité supérieure à $1 \%\left(\chi^{2}>160\right.$ pour 16 degrés de liberté).

Il existe par conséquent une liaison hautement significative entre intensité du dépérissement du pin d'origine portugaise et type de sols, donc hydromorphie. Le dépérissement est maximum dans les stations les plus hydromorphes.

\subsection{Ensemble de la zone où sévit le dépérissement}

\subsection{Comportement du Pin landais}

Ultérieurement, au cours de notre enquête, nous avons pu vérifier que le dépérissement n'affectait pas le pin d'origine locale, au moins dans la mesure où nous n'a- 


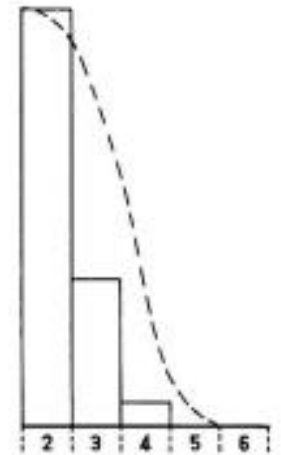

Peuplement IV

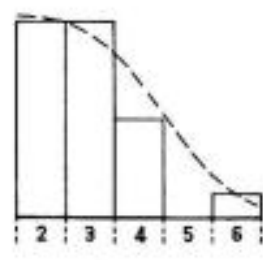

Peuplement III

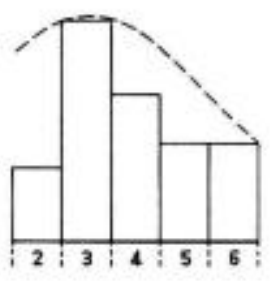

Peuplement II
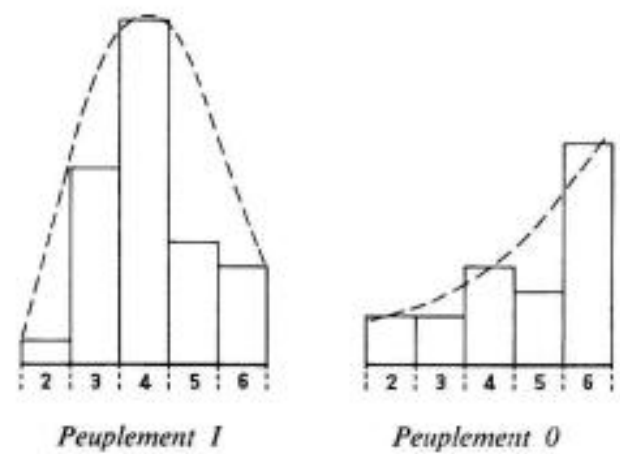

$\leq$

四

学

c

$-$

욤

䎡

T1

而

3

Fic. 3. - Intensité du dépérissement et types de sols (sols de type 2, 3, 4, 5 et 6)

 
vons pas fait d'erreurs sur l'origine, quel que soit le type de sol. Nous n'avons observé des difficultés de croissance et quelques signes voisins des signes de dépérissement que dans les zones très hydromorphes, caractérisées par des sols à hydromor de $1 \mathrm{mè}-$ tre d'épaisseur ou plus.

Le pin d'origine landaise n'est donc pas affecté de manière visible par l'hydromorphie, sauf dans des cas très extrêmes.

\subsection{Comportement du Pin portugais}

Les résultats obtenus par une étude détaillée d'une zone restreinte pouvaient ne pas être applicables à l'ensemble de la zone atteinte par le dépérissement. Pour vérifier si la liaison observée entre le dépérissement et I'hydromorphie était généralisée, nous avons effectué une étude point par point sur 28 autres propriétés, réparties sur l'ensemble de la Grande Lande, en comparant pour chacun de ces contrats les sols des zones très atteintes, moyennement atteintes et peu atteintes.

Nous avons ainsi pû dresser un graphique de la répartition des sols en fonction du type de peuplement (fig. 3). Pour chaque type de sol, les différents types de peuplement sont représentés par un bloc diagramme dont la hauteur est fonction du nombre d'observations.

Dans ce graphique, n'apparaissent pas les peuplements notés V et VI. Ils sont en effet très peu représentés et correspondent le plus souvent à des conditions particulières : zone enrichie artificiellement, zone limitrophe des Landes.

De même les sols de type $1 \mathrm{n}$ 'apparaissent pas, car deux observations seulement ont pu y être effectuées.

Ces réserves étant faites nous obtenons une distribution tout à fait remarquable. Aux peuplements les plus atteints correspondent les sols les plus hydromorphes.

Pour cet ensemble d'observations nous pouvons également utiliser le test du $\chi^{2}$. Nous obtenons un $\chi^{2}$ de $62,4($ d.d.l. $=26$ ) et par conséquent encore nettement supérieur au seuil de la table au risque de $1 \%$.

Il est donc possible d'extrapoler les résultats obtenus à l'ensemble des peuplements en voie de dépérissement : il existe une liaison très significative entre l'intensité du dépérissement et lintensité de l'hydromorphie.

\subsection{Anomalies dans le comportement du Pin d'origine portugaise}

La localisation des zones maximales de dépérissement dans la propriété RAsson par exemple présente quelques anomalies.

Dans la partie sud de la propriété où l'hydromorphie est la plus marquée, le long des fossés de drainage, le dépérissement est d'autant plus accentué que les pins sont situés plus loin des fossés de drainage, bien que morphologiquement le sol ne varie pas.

D'autre part, au centre de cette propriété existe un ancien champ cultivé où il est possible d'observer côte à côte des Pins des deux origines. Leur croissance 
est tout à fait semblable et remarquable, comparée à celle des Pins situés en dehors de cette zone. En particulier la note attribuée aux Pins portugais est de V ou VI, alors que en dehors de cette zone la note $\mathrm{n}^{*} \mathrm{a}$ jamais dépassée IV, et bien que cette ancienne zone cultivée soit parfois très hydromorphe avec des sols de type 6 .

Dans une autre propriété, PEREIRE, il n'existe pas non plus de liaison entre hydromorphie et dépérissement. Ces quelques observations nous amènent à penser que si l'hydromorphie est un facteur déterminant, les conditions de nutrition minérale pourraient jouer un rôle important dans le déterminisme du dépérissement du Pin d'origine portugaise.

Deux voies sont possibles pour déterminer le rôle des conditions de nutrition : recherche des liaisons entre propriétés chimiques des sols et dépérissement d'une part, recherche des liaisons entre l'absorption des éléments minéraux et intensité du dépérissement par analyses foliaires.

Nous avons simultanément utilisé ces deux possibilités. Pour des raisons de commodité de raisonnement, nous exposerons tout d'abord les résultats obtenus par analyses foliaires.

\section{3. - ANALYSES FOLIAIRES}

\section{1. - Introduction - Premiers résultats}

Avant même une étude détaillée des sols, quelques prélèvements d'aiguilles, destinés à avoir une idée approximative du niveau de nutrition, furent effectués dès l'hiver 1964-65, dans des peuplements portugais dépérissants et dans des peuplements landais voisins. Le nombre d'échantillons étudiés étant toutefois trop faible pour permettre d'avoir une certitude, une nouvelle campagne de prélèvements et d'analyses fut effectuée pendant l'hiver 1965-66. Elle portait sur 90 échantillons, représentant chacun un mélange des aiguilles de 5 pins voisins, portugais ou landais, et permit d'aboutir aux résultats suivants :

a) les Pins portugais avaient en effet une nutrition très faible en $\mathrm{P}_{2} \mathrm{O}_{5}, \mathrm{~N}, \mathrm{~K}$, $\mathrm{Mn}$ et $\mathrm{Cu}$. On ne trouvait de peuplements indemnes que s'ils étaient bien alimentés, particulièrement en $\mathrm{P}_{2} \mathrm{O}_{5}$ (plus de $0,30 \%$ dans les aiguilles);

b) les Pins landais bien qu'indemnes de tout signe de dépérissement avaient des aiguilles aussi pauvres en éléments minéraux que les Pins portugais atteints;

c) deux reboisements très étendus, la propriété RAsson dont il a déjà été question et la propriété PEREIRE en Gironde avaient pu permettre de prélever des échantillons suffisamment nombreux pour étudier des corrélations entre la teneur en divers éléments minéraux et une notation subjective de l'état des peuplements. Pour certains éléments la corrélation existait dans ces deux reboisements : $\mathrm{P}_{2} \mathrm{O}_{5}$ (très significative), $\mathrm{K}$ (très significative) $\mathrm{N}$ (faiblement significative)

d) pour d'autres éléments, $\mathrm{Ca}, \mathrm{Mn}, \mathrm{Cu}$, on ne mit en évidence une corrélation que dans l'un ou l'autre de ces deux peuplements; 
e) la teneur en magnésium était toujours suffisante et sans relation avec l'intensité du dépérissement.

Ainsi commençaient à se confirmer deux des hypothèses avancées, l'explication génétique et l'explication nutritionnelle. Cependant, l'interprétation de cette deuxième série d'analyses foliaires restait incomplète du fait de l'absence d'un système d'appréciation objectif et chiffré de l'état des arbres. La mise au point d'une formule de notation, faisant intervenir diverses caractéristiques des peuplements, a permis d'envisager en 1966-67 une troisième campagne de prélèvements dont le but était à la fois de contrôler et de préciser les interprétations qui avaient été faites les années précédentes.

\section{2. - Choix et mise au point d'une notation}

Devant l'ambiguïté quasi constante pour préciser l'intensité du dépérissement d'un peuplement et afin de mieux situer cette intensité par rapport à d'autres, il s'est avéré nécessaire de disposer d'un outil à la fois plus précis et moins subjectif.

L'examen des peuplements dépérissants permettait de dissocier deux groupes de caractéristiques :

- celles tenant à la forme même de l'arbre (décroissance rapide, tige fine) et à la densité et la régularité du peuplement lui-même ;

- celles relevant de l'aspect de la masse végétale active : mauvais état des aiguilles, longueur réduite, disparition plus ou moins rapide de celles âgées de plus d'un an, toutes ces caractéristiques donnant au peuplement un aspect clair.

\subsection{Formule élémentaire}

Pour faire apparaître ces divers facteurs, le premier groupe de caractéristiques apparaît dans la formule élémentaire : $\frac{h \times C^{2} \times 10}{a} \times d$

$h$ : hauteur moyenne en mètres,

$C$ : circonférence moyenne à 1,30 en mètres,

$a$ : âge du peuplement en années,

$d$ : coefficient de densité du peuplement, coefficient défini suivant le tableau 1.

En raison du changement d'équilibre introduit par l'éclaircie entre les arbres, il nous a semblé nécessaire de distinguer les peuplements ayant été éclaircis de ceux qui ne l'ont pas été, ou qui l'ont été à une période trop éloignée ; en effet dans le premier cas l'hétérogénéité du peuplement subsistant malgré les corrections apportées par l'éclaircie justifie l'utilisation d'un coefficient plus sévère.

Dans le cas de quelques Pins épars, même si lors d'une éclaircie il y a eu intervention dans la zone considérée, il serait illogique d'introduire un coefficient différent à cause de ce traitement. 
TABLEAU 1

Coefficient de densité

\begin{tabular}{|c|c|c|}
\hline & $\begin{array}{l}\text { Peuplement } \\
\text { eclairci }\end{array}$ & $\begin{array}{l}\text { Peuplement } \\
\text { non éclairci }\end{array}$ \\
\hline 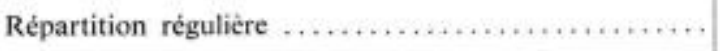 & 1 & 1 \\
\hline 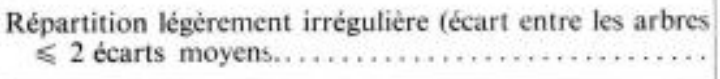 & 0,75 & 0,90 \\
\hline Ecart $<2$ écarts moyens : vides, bouquets ........... & 0,50 & 0,70 \\
\hline Quelques Pins épars ......... & - & 0,20 \\
\hline
\end{tabular}

\section{TABleau 2}

Note attribuée à chaque verticille pour Tobtention de la note $\mathrm{E}$

\begin{tabular}{|c|c|c|}
\hline & $\begin{array}{l}\text { Verticilles } \\
\text { complets }\end{array}$ & $\begin{array}{l}\text { Verticilles } \\
\text { incomplets }\end{array}$ \\
\hline $\begin{array}{l}\text { Verticille d'un an avec aiguilles d'un an en nombre } \\
\text { suffisant }\end{array}$ & 1 & 0,5 \\
\hline $\begin{array}{l}\text { Verticille d'un an avec quelques aiguilles d'un an (petit } \\
\text { plumct) } \ldots \ldots \ldots \ldots \ldots \ldots \ldots \ldots \ldots \ldots \ldots \ldots \ldots \ldots \ldots\end{array}$ & 0 & 0 \\
\hline $\begin{array}{l}\text { Ou } 2^{e} \text { ou } 3^{e} \text { verticille d'un an (pousses d'août) peu } \\
\text { développé } \ldots \ldots \ldots \ldots \ldots \ldots \ldots \ldots \ldots \ldots \ldots \ldots \ldots \ldots \ldots \ldots \ldots\end{array}$ & 0,5 & 0 \\
\hline Verticilles de deux ans avec aiguilles de deux ans ...... & 1 & 0,5 \\
\hline $\begin{array}{l}\text { Verticilles de deux ans avec aiguilles d'un an en nombre } \\
\text { suffisant } \quad \ldots \ldots \ldots \ldots \ldots \ldots \ldots \ldots \ldots \ldots \ldots \ldots \ldots \ldots\end{array}$ & 0,5 & 0 \\
\hline $\begin{array}{l}\text { Verticilles de deux ans avec quelques aiguilles d'un an } \\
\text { (petit plumet) } \ldots \ldots \ldots \ldots \ldots \ldots \ldots \ldots \ldots \ldots \ldots \ldots\end{array}$ & 0 & 0 \\
\hline Verticilles de trois ans avec aiguilles de trois ans........ & 1,5 & 1 \\
\hline Verticilles de trois ans avee aiguilles de deux ans $\ldots . .$. & 1 & 0,5 \\
\hline $\begin{array}{l}\text { Verticilles de trois ans avec aiguilles d'un an en nombre } \\
\text { suffisant }\end{array}$ & 0,5 & 0 \\
\hline $\begin{array}{l}\text { Verticilles de trois ans avec quelques aiguilles d'un an } \\
\text { (petit plumet) }\end{array}$ & 0 & 0 \\
\hline
\end{tabular}

Notes : Verticille complet $=$ répartition symétrique des rameaux, il est admis cependant qu'il puisse manquer un rameau sur l'ensemble des rameaux du verticille, exemple ; cas de pins en bouquet.

Il n'est pas tenu compte de l'élagage. 
Cette formule élémentaire équivaut à une vitesse moyenne d'accroissement annuel par unités de surface.

\subsection{Note $\mathrm{E}$}

La notation de l'aspect de la masse végétale (note $E$ ) s'obtient par sommation des cotations de chaque verticille (flèche non comprise), selon le tableau 2 .

Primitivement, un coefficient sanitaire était prévu. En fait, des essais en collaboration avec le service d'Entomologie ont fait ressortir qu'au niveau du but recherché l'aspect des verticilles et des aiguilles intègre l'état sanitaire de l'arbre.

Cette note $E$ équivaut à un potentiel énergétique de l'arbre : masse relative de tissus actifs (photosynthése), niveau des réserves (métabolisme).

Des notations récentes sur peuplements fertilisés au dépérissement freiné ou stoppé, indique l'intérêt qu'il y aurait à introduire la longueur moyenne des aiguilles des premier et deuxième verticilles pour serrer de plus près l'aspect « revigoré $n$ d'un peuplement.

\subsection{Note globale}

Cette note globale est une combinaison de la note élémentaire et de la note $E$. Nous avons utilisé le produit de ces deux notes, ce qui donne pour la note globale la formule suivante :

$$
\frac{E \times h \times C^{2} \times 10 \times d}{a}
$$

L'étude de l'erreur sur la note globale (les mesures caractérisant un peuplement portant sur 50 arbres) donne une erreur théorique maximale de $6 \%$, ce qui autorise le calcul de la note globale sur les moyennes de chaque facteur. Cependant, dans le cas où la zone caractéristique du peuplement renfermerait des arbres de tailles très diverses, il faudrait revenir à la moyenne de notation globale de chaque arbre.

L'amplitude de variation de la note globale sur peuplements dépérissants et non dépérissants a été jusqu'à présent comprise entre 0,5 et 40 pour des arbres d'âge compris entre 10 et 20 ans.

\section{3. - Résultats de la campagne de prélèvements 1966-1967}

\subsection{Choix et notation du peuplement et des arbres échantillonnés}

La campagne de prélèvements 66-67 avait essentiellement pour but :

a) l'étude et la comparaison dans des conditions de sol et de pédoclimat semblables, des niveaux de nutrition de peuplements d'origine autochtone "landais » et de peuplements allochtones qui seront dénommés " portugais » en raison d'un 
faisceau de présomptions suffisamment convergent : années des semis, provenance presque générale des semences portugaises aux mêmes époques ;

b) une tentative de dissociation plus précise des origines par dosages des $\alpha$ et $\beta$ pinénes dans les produits de microgemmage, tentative n'ayant pas donné de résultats nets en raison des évolutions des gemmes durant leur période de prélèvements mais dessinant cependant des tendances qui justifieraient la reprise de cette étude par un service d'amélioration des plantes.

\subsection{Recherche des couples}

Afin d'éliminer toute modification de sol ou de drainage, les couples étaient dans la mesure du possible recherchés dans deux peuplements contigus : conditions réalisées rigoureusement à Pallas, Dussillol Sud. Lorsque les deux peuplements ne réalisaient pas cette condition, la végétation naturelle servait de critère de comparaison.

\subsection{Recherche des arbres}

A l'intérieur du peuplement après prospection de celui-ci, une zone représentative, tant du point de vue végétation naturelle que du point de vue aspect des arbres et densité du peuplement, était définie ; à l'intérieur de cette zone, cinq arbres étaient choisis assez rapprochés d'une part, représentatifs d'autre part des arbres courants du peuplement. Chacun était noté ensuite séparément, la note globale moyenne étant obtenue à partir des notes globales indivuelles.

Il faut noter cependant pour le cas de Rasson-expérience que les impératifs techniques du microgemmage imposaient un diamètre minimal et que les Pins ainsi choisis représentent un échantillonnage de caractéristiques supérieures aux moyennes de chacun des facteurs.

Le tableau 3 donne la liste des peuplements choisis et les caractéristiques moyennes des arbres étudiés dans chacun d'eux.

\subsection{Corrélations, dépérissement, nutrition}

Par une analyse statistique rigoureuse effectuée avec l'aide de la Station de Biométrie du C.N.R.F., nous avons cherché, pour les peuplements d'origine portugaise seulement, à relier le niveau de nutrition en divers éléments et l'état de vigueur des arbres.

Les mêmes techniques d'analyses statistiques que lors de notre première étude (campagne de prélèvements 1965-1966) ont été utilisées : calcul de coefficients de corrélation totale entre variables à expliquer et variables explicatives, calcul de ces mêmes coefficients entre variables explicatives, et enfin, analyse de régression progressive.

Les variables à expliquer sont au nombre de 6 : pousse moyenne annuelle, accroissement annuel moyen sur le diamètre, longueur moyenne des aiguilles, note $E$, 
TABleaU 3

Liste et caractéristiques des peuplements éfudièr

\begin{tabular}{|c|c|c|c|c|c|c|c|}
\hline Lieu & Origine & Age & $\begin{array}{l}\text { Hauteur } \\
\text { moy. } \\
\text { en m }\end{array}$ & $\begin{array}{c}\text { Circonf. } \\
\text { moy. } \\
\text { en } \mathrm{m} \\
\text { a } 1,30 \mathrm{~m}\end{array}$ & $\begin{array}{l}\text { Longueur } \\
\text { moyenne } \\
\text { des } \\
\text { aiguilles } \\
\text { (cm) }\end{array}$ & $E$ & $\begin{array}{c}\text { Note } \\
\text { globale }\end{array}$ \\
\hline Mimizan & landaise & & & & & & \\
\hline Témoin & Bloc I & 10 & 4,38 & 0,220 & 19,8 & 5,4 & 1,19 \\
\hline- & Bloc II & 10 & 4,66 & 0.250 & 22,2 & 5,6 & 1,87 \\
\hline - & Bloc III & 10 & 4,30 & 0,214 & 20,8 & 5,4 & 1,08 \\
\hline \multirow{3}{*}{$\begin{array}{l}\text { Luxey } \\
\text { "La Maison " } \\
\text { "Borde } "\end{array}$} & & & & & & & \\
\hline & landaise & 14 & 8,48 & 0,446 & 20,4 & 7,1 & 8,71 \\
\hline & landaise ? & 14 & 9,56 & 0,438 & 20,3 & & \\
\hline Herré & portugaise & 15 & 9,00 & 0,478 & 23,8 & 8,6 & 13,71 \\
\hline \multirow[t]{2}{*}{ Liposthey } & landaise & 20 & 13,00 & 0.564 & 17,8 & 7,4 & 16,25 \\
\hline & portugaise & 12 & 5,32 & 0,260 & 16,6 & 5,6 & 1,70 \\
\hline \multirow[t]{2}{*}{ Pissos } & landaise & 20 & 14,68 & 0.666 & 18,8 & 9,4 & 30,68 \\
\hline & portugaise & 10 & 6,26 & 0,350 & 18,8 & 6,0 & 3,52 \\
\hline \multirow{3}{*}{$\begin{array}{r}\text { Dussillol } \mathrm{N} \\
\mathrm{S}\end{array}$} & landaise? & 14 & 9,24 & 0,402 & 18,8 & 5,5 & 6,07 \\
\hline & landaise & 14 & 9.56 & 0,464 & 18,4 & 8,1 & 12,28 \\
\hline & portugaise & 11 & 6,74 & 0,363 & 18,9 & 5,7 & 3,78 \\
\hline \multirow[t]{2}{*}{ Pallas } & landaise & 15 & 12,30 & 0,646 & 18,6 & 9,0 & 30,40 \\
\hline & portugaise & 12 & 7,18 & 0,366 & 19.8 & 6,1 & 5,46 \\
\hline \multirow{2}{*}{$\begin{array}{l}\text { Rasson } \\
\text { champ } \\
\text { expérience } \\
\text { témoins }\end{array}$} & portugaise & 12 & 9,30 & 0,471 & 19,9 & 5,93 & 10,98 \\
\hline & portugaise & 14 & 5,13 & 0,256 & 17,9 & 4,9 & 0,62 \\
\hline
\end{tabular}

note globale de novembre 1966 et note globale de janvier - février 1967, ces deux dernières variables étant une combinaison faisant intervenir les trois premières.

Les variables explicatives sont au nombre de 8 . Ce sont 8 éléments totaux dosés dans les aiguilles après séchage à l'étuve à $65^{\circ}$ et minéralisation complète soit par voie humide à l'aide de $\mathrm{H}_{2} \mathrm{SO}_{4}$ et $\mathrm{H}_{2} \mathrm{O}_{2}$ pour $\mathrm{N}, \mathrm{P}_{2} \mathrm{O}_{5}, \mathrm{~K}, \mathrm{Ca}, \mathrm{Mg}, \mathrm{Mn}$ et $\mathrm{Cu}$, soit par voie sèche pour $\mathrm{B}$. Ces 8 éléments ont été dosés de la manière suivante :

$\mathrm{N}$ distillation au micro-Kjeldahl Parnas-Wagner ;

$\mathrm{P}_{2} \mathrm{O}_{5}$ colorimétrie au molybdate d'ammonium et réduction au chlorure stanneux ;

$\mathrm{K}$ et $\mathrm{Ca}$ spectrophotométrie de flamme ;

$\mathrm{Mg} \quad$ colorimétrie au jaune de titane ;

$\mathrm{Mn}$ colorimétrie après oxydation de l'ion $\mathrm{Mn}^{++}$en $\mathrm{MnO}_{4}$ - par le périodate de potassium ; 
$\mathrm{Cu} \quad$ colorimétrie par le diéthyldithiocarbamate de sodium ;

B colorimétrie à la dianthrimide 1-1'.

Les résultats sont donnés au tableau 4.

\subsection{Note globale janvier-février 1967}

Les résultats obtenus pour les deux notes globales de 1966 et de 1967 ne diffèrent pas notablement, nous ne raisonnerons que sur l'une d'elle, la dernière attribuée, celle de janvier-février 1967.

Cinq éléments ont un coefficient de corrélation très significatif avec la note globale de 1967 (tableau 5). Ce sont, dans l'ordre, le phosphore, le potassium, l'azote, le cuivre et le manganèse. Les trois éléments majeurs apparaissent done en tête, le phosphore ayant la liaison la plus significative suivi de près par le potassium.

Si nous rapprochons ces résultats de ceux obtenus lors de la campagne 1965-1966 en utilisant le premier système de notation, nous constatons qu'ils sont extrêmement voisins. Nous avions en effet mis l'accent sur le rôle du phosphore et du potassium et à un degré moindre et moins fréquemment sur celui de deux oligoéléments, le cuivre et le manganèse.

Une différence apparait cependant : en 1965-1966 nous n'avions pu mettre en évidence qu'un faible rôle de l'azote, tout en soulignant son taux extrêmement faible dans les aiguilles. Or, au cours de la campagne 1966-1967, il apparait une liaison significative entre intensité du dépérissement et teneur en azote des aiguilles.

Les résultats de l'analyse de régression progressive sont schématisés par la figure 4-A.

La première variable introduite est le phosphore qui permet d'expliquer plus de $60 \%$ de la variation totale (coefficient de corrélation multiple $=0,623$ ). En fin de régression, le coefficient de corrélation multiple est de 0,729 et deux éléments restent significatifs : le phosphore ( $T$ Student $=2,27$ ) et le potassium $(T=2,23)$, ce qui nous confirme l'importance de ces deux éléments dans le dépérissement.

Note globale janvier-février 1967 ou ancienne notation de 1965, permettent donc d'arriver aux mêmes conclusions. Ces deux types de notation de l'intensité du dépérissement s'appliquent en fait à un ensemble de facteurs que l'on peut décomposer ainsi : ralentissement de la croissance, diminution de la densité du peuplement par mortalité, diminution de la masse foliaire et chute des aiguilles.

II nous a paru intéressant de décomposer le phénomène du dépérissement et de chercher à lier les différentes composantes à la nutrition.

\subsection{Croissance en hauteur et croissance en diamètre}

Les peuplements portugais que nous avons étudiés étant tous d'âge très comparable, compris entre 10 et 15 ans, nous avons pris comme critère de croissance l'accroissement moyen annuel en hauteur et l'accroissement moyen annuel sur le diamètre. 


\section{TABLEAU 4}

Composition foliaire moyenne des peuplements étudiés ; origines landaises et portugaises

\begin{tabular}{|c|c|c|c|c|c|c|c|c|}
\hline Origine & $\begin{array}{l}\mathrm{N} \\
\%\end{array}$ & $\underset{\%}{\mathrm{P}_{2} \mathrm{O}_{3}}$ & $\begin{array}{l}\mathrm{K} \\
\%\end{array}$ & $\mathrm{Ca}$ & $\underset{\%}{\mathrm{Mg}}$ & $\mathrm{Mn}_{\%}$ & $\begin{array}{c}\mathrm{Cu} \\
\text { p.p.m. }\end{array}$ & $\begin{array}{c}\text { B } \\
\text { p.p.m. }\end{array}$ \\
\hline $\begin{array}{c}\text { Potugaise } \\
\text { Rasson }\end{array}$ & 0,90 & 0,17 & 0,38 & 0,16 & 0,18 & 0,07 & 4,3 & 19,6 \\
\hline $\begin{array}{l}\text { Rasson } \\
\text { ancien } \\
\text { champ }\end{array}$ & 1,08 & 0,31 & 0,54 & 0.11 & 0.14 & 0,46 & 9,6 & 22,3 \\
\hline Pissos & 0,88 & 0,17 & 0,47 & 0,16 & 0,17 & 0,08 & 4,5 & 27,4 \\
\hline Liposthey & 0,74 & 0,18 & 0,42 & 0,14 & 0,13 & 0,06 & 6,3 & 26,1 \\
\hline Pallas & 0,91 & 0,22 & 0,44 & 0,18 & 0,16 & 0,05 & 4,9 & 17,7 \\
\hline Luxey & 1,29 & 0,17 & 0,39 & 0.14 & 0,16 & 0,06 & 3,1 & 26,6 \\
\hline Dussillol & 0,98 & 0,27 & 0,44 & 0.16 & 0,12 & 0,05 & 5,2 & 23,1 \\
\hline Herré & 1,17 & 0,29 & 0,55 & 0,16 & 0,12 & 0,06 & 6,7 & 18,2 \\
\hline $\begin{array}{l}\text { Landaise } \\
\text { Pissos }\end{array}$ & 0,96 & 0,20 & 0.45 & 0,18 & 0,21 & 0,10 & 8,4 & 29,1 \\
\hline Liposthey & 0,77 & 0,18 & 0,44 & 0,17 & 0,11 & 0,09 & 5,6 & 28,6 \\
\hline Pallas & 0,79 & 0,23 & 0.47 & 0,17 & 0,18 & 0,06 & 6,2 & 27,4 \\
\hline Dussillol & 1,04 & 0,33 & 0,56 & 0,19 & 0,15 & 0,06 & 5,0 & 23,8 \\
\hline $\begin{array}{l}\text { Mimizan } \\
\text { (témoins) }\end{array}$ & 1,24 & 0.22 & 0,40 & 0.17 & 0,12 & 0,08 & 6,7 & 28,4 \\
\hline
\end{tabular}

TABLEAU 5

Coefficients de corrélation totale (* significatifs au risque de I\%)

\begin{tabular}{|c|c|c|c|c|c|c|c|c|}
\hline & $\mathrm{N}$ & $\mathrm{P}_{2} \mathrm{O}_{6}$ & K & $\mathrm{Ca}$ & $\mathrm{Mg}$ & $\mathrm{Mn}$ & $\mathrm{Cu}$ & B \\
\hline Note globale 1967 & $0,42^{*}$ & $0,62^{*}$ & $0,55^{*}$ & 0,15 & 0,22 & $0,40^{*}$ & $0,42^{\circ}$ & 0,17 \\
\hline $\begin{array}{l}\text { Pousse moyenne } \\
\text { annuelle } \ldots . . .\end{array}$ & $0,42^{*}$ & $0,60^{*}$ & $0,50^{+}$ & $0,36^{*}$ & 0,32 & $0,56^{*}$ & $0,58^{*}$ & 0,24 \\
\hline $\begin{array}{l}\text { Accroissement } \\
\text { moyen annuel } \\
\text { sur le diamétre } \\
\text { à } 1,30 \mathrm{~m}\end{array}$ & $0,37^{*}$ & $0,62^{*}$ & $0.54^{*}$ & $-0,23$ & $-0,29$ & $0,53^{*}$ & $0,51^{*}$ & 0,28 \\
\hline $\begin{array}{c}\text { Longueurs des ai- } \\
\text { guilles ......... }\end{array}$ & $0,50^{*}$ & $0,37^{*}$ & $0,39^{*}$ & 0,07 & 0,28 & 0,10 & $-0,32$ & $-0,05$ \\
\hline Note $E \quad \ldots \ldots \ldots$ & $0,42^{*}$ & $0,37^{*}$ & $0,45^{*}$ & 0,08 & $-0,31$ & 0,05 & 0,25 & 0,11 \\
\hline
\end{tabular}



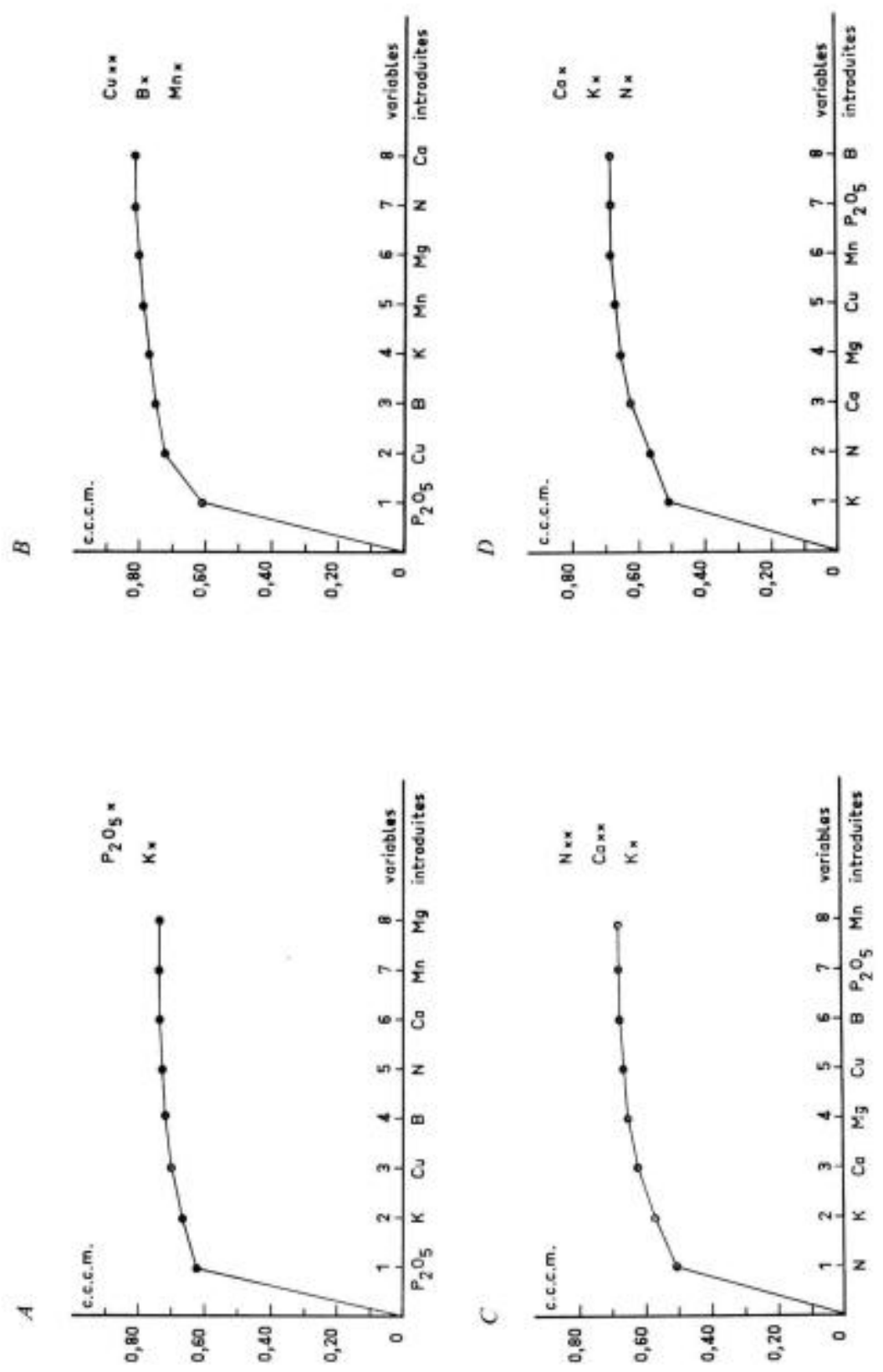

FIG. 4. - Analyse de régression multiple :

A. Note globale $1967 ;$ B. Pousse moyenne annuelle: C. Longateur des aiguilles; D. Note E. (c.c.c.m. : carré du coefficient de corrélation multiple. Variables significatives en fin de régression au seuil de $1 \% * *$, au seuil de $5 \%{ }^{*}-$ ). 
Les résultats obtenus pour ces deux formes d'accroissement sont extrêmement voisins.

\section{a) Croissance en hauteur}

Cinq éléments ont un coefficient de corrélation totale très significatif avec la croissance en hauteur. Ce sont les mêmes qui intervenaient pour la note globale, à savoir $\mathrm{N}, \mathrm{P}_{2} \mathrm{O}_{5}, \mathrm{~K}, \mathrm{Cu}$ et $\mathrm{Mn}$. De la même façon, c'est le phosphore qui a le coefficient de corrélation totale le plus élevé, mais des trois éléments majeurs, c'est le seul qui arrive en tête. Le phosphore est en effet suivi de très près par le cuivre et le manganèse $(r=0,58$ et 0,56$)$.

L'importance de ces deux oligoéléments est encore soulignée par les résultats de l'analyse de régression progressive. Si le phosphore introduit en tête permet d'expliquer encore $60 \%$ de la variation totale, en fin de régression $(r=0,803)$ seuls les trois oligoéléments, cuivre, bore et manganèse restent significatifs (fig. 4-B).

\section{b) Croissance en diamètre}

Comme nous l'avons déjà dit, les résultats sont extrêmement voisins de ceux obtenus pour la croissance en hauteur. Quelques différences cependant apparaissent. Le potassium semble jouer un rôle un peu plus important que pour la croissance en hauteur. Par son coefficient de corrélation totale, cet élément arrive en seconde position derrière le phosphore et donc avant les deux oligoéléments : manganèse et cuivre. En fin de régression, d'autre part, le potassium reste significatif avec le bore et le cuivre.

D'autre part, le calcium qui avait un coefficient de corrélation totale légèrement significatif avec la croissance en hauteur n'apparaît plus ici.

\subsection{Longueur des aiguilles}

Nous pouvons supposer que la longueur des aiguilles est une estimation très approchée de la production de matière verte, ou que du moins il existe une liaison entre la longueur des aiguilles et la production totale de matière verte.

Les résultats que nous obtenons sont extrêmement différents de ceux obtenus pour la production de bois.

En effet, trois éléments seulement ont un coefficient de corrélation totale significatif avec la longueur des aiguilles. Ce sont uniquement les trois éléments majeurs $\mathrm{N}, \mathrm{K}$ et $\mathrm{P}_{2} \mathrm{O}_{5}$, l'azote arrivant assez nettement en tête. Il est connu en effet, depuis très longtemps que l'azote est un facteur essentiel de production de matière verte. Dans l'analyse de régression progressive, l'introduction des trois éléments $\mathrm{N}, \mathrm{K}$ et Ca permet d'expliquer $60 \%$ de la variation.

Un fait remarquable est la disparition quasi complète des oligoéléments qui jouaient un rôle majeur dans la production de bois. Leur coefficient de corrélation totale n'est pas significatif et d'autre part ils n'y apparaissent plus en fin de régression, 
où restent très significatifs l'azote avec un $T$ très élevé $(T=3,12)$, le calcium et le potassium (fig. 4-C).

\subsection{Note E}

Cette note traduit la persistance des aiguilles. II existe une analogie certaine entre les résultats obtenus pour la longueur des aiguilles et les résultats obtenus pour cette note $E$ qui s'applique à une des composantes importantes du dépérissement.

Comme précédemment, seuls les trois éléments majeurs ont un coefficient de corrélation totale significatif, les oligoéléments n'intervenant pas. Mais catte fois le potassium arrive en tête devant l'azote puis le phosphore. Dans l'analyse de régression progressive, l'introduction des trois éléments N, K, Ca permet d'expliquer $60 \%$ de la variation. En fin de régression restent significatifs le calcium, le potassium et enfin l'azote, les oligoéléments n'intervenant toujours pas (fig. 4-D).

L'analogie est donc certaine avec les résultats relatifs à la longueur des aiguilles avec cependant une légère différence. Ce sont en effet deux cations, le potassium et le calcium, qui se révèlent être plus que l'azote les facteurs les plus importants.

Il est intéressant d'essayer de rechercher une explication à cet état de fait. De l'avis des forestiers landais, le dépérissement et en particulier la chute des aiguilles, qui en est un des aspects principaux, s'est toujours manifesté avec le plus d'amplitude après les années de grand froid.

Il n'est pas en effet étonnant de penser que le Pin portugais d'origine méridionale, soit sensible au froid. Or, la persistance des aiguilles est fonction de deux éléments le potassium et le calcium, dont l'un, le potassium, détermine en partie la résistance au froid. Il est impossible d'affirmer qu'il existe une telle relation de cause à effet, mais la question mérite d'être posée.

\subsection{Récapitulation des résultats}

Les conclusions auxquelles nous avions abouti en 1965-1966 en utilisant l'ancien système de notation sont pleinement confirmées par les résultats obtenus en 1966-1967 en utilisant un système de notation beaucoup plus objectif (note globale 1967).

Nous pouvons ainsi résumer ces résultats :

- rôle déterminant du phosphore et à un degré moindre du potassium dont les déficiences constituent la cause principale du dépérissement du Pin maritime d'origine portugaise ;

- rôle non négligeable de deux oligoéléments le cuivre et le manganèse.

Enfin l'azote apparaît nettement dans les corrélations entre dépérissement et nutrition alors qu'en 1965-1966, cettte corrélation n'apparaissait que très faiblement, le taux d'azote étant partout très faible.

La campagne 1966-67 nous permet cependant d'aller beaucoup plus loin que 
celle de 1965-1966 gâce à l'étude des relations entre les diverses composantes du dépérissement et la nutrition.

La diminution de croissance en hauteur et en diamètre, donc la baisse de production, dépend surtout des déficiences en phosphore et également en oligoéléments, cuivre, manganèse et bore, ces trois oligoéléments étant seuls significatifs en fin de régression.

La production de matière verte approchée par la mesure de la longueur des aiguilles dépend surtout des trois éléments majeurs, N, P et K, l'azote jouant le rôle fondamental, alors que les oligoéléments n'interviennent plus.

La persistance des aiguilles est également sous la dépendance de la nutrition en ces trois éléments majeurs, les oligoéléments n'y jouant toujours aucun rôle. Néanmoins, le potassium et le calcium semblent les deux éléments essentiels dans le déterminisme de la chute des aiguilles.

\subsection{Comparaison entre Pins landais et Pins portugais}

Les différentes observations effectuées en 1965-1966 laissaient supposer que les exigences des deux origines n'étaient pas identiques.

Nous avions pu montrer qu'en station naturellement riche ou enrichie artificiellement, la nutrition était très comparable entre Pins landais et Pins portugais : le niveau de nutrition en phosphore et en potassium était élevé pour les deux origines : 0,60 à $0,70 \%$ pour le potassium, 0,35 à $0,40 \%$ pour le phosphore.

Dans ces conditions de nutrition, aucun signe de dépérissement n'apparaissait quelle que soit l'origine.

En station pauvre, ce qui est le cas le plus fréquent, la nutrition des deux origines est encore assez semblable, mais très déficiente. Les teneurs en $\mathrm{N}, \mathrm{P}_{2} \mathrm{O}_{5}$ et $\mathrm{K}$ s'abaissaient en 1965-1966 respectivement à moins de $1 \%$ pour $\mathrm{N}, 0,20$ à $0,25 \%$, pour $\mathrm{P}_{2} \mathrm{O}_{5}$ et 0,30 à $0,40 \%$ pour $\mathrm{K}$.

Mais si la nutrition est aussi mauvaise pour les deux origines, leur comportement devient différent. Avec un niveau de nutrition très bas, les peuplements de Pins landais, s'ils ont une croissance ralentie, ne présentent pas de signe de dépérissement.

Par contre, pour un niveau identique, les Pins portugais présentent des troubles graves pouvant aller jusqu'à la disparition complète du peuplement.

Nous avons essayé au cours de la campagne 1966-1967 de démontrer le bien fondé de cette hypothèse de plus grande exigence du Pin portugais par rapport au Pin landais, hypothèse basée sur un faible nombre d'analyses et non appuyée sur des tests statistiques.

Deux voies étaient possibles pour confirmer ou infirmer cette hypothèse. Nous pouvions en effet procéder soit expérimentalement soit par enquête sur le terrain. Nous avons choisi ce second procédé bien que des résultats irréfutables soient beaucoup plus difficiles à obtenir ainsi ; mais ce moyen avait l'avantage d'être rapide et de nous permettre de travailler sur des arbres de 10 à 15 ans et non sur des jeunes semis dont le comportement est différent. 
Nous avons choisi quatre couples de peuplements des deux origines situées côte à côte ou dans des conditions aussi voisines que possible et en conditions normales, donc sur sols pauvres. Ces couples sont situés sur quatre communes du département des Landes : Pissos, Liposthey, Retjons (Propriété Pallas) et Bouriot (Propriété Dussillol). Mais il est bien évident que les conditions de sols ne peuvent être rigoureusement identiques à l'intérieur de chaque couple.

Pour chacun des peuplements, nous avons procédé au prélèvement de cinq échantillons d'aiguilles, chaque échantillon correspondant à un pin étant analysé séparément. Nous avons donc quatre couples de deux fois cinq échantillons chacun (5 d'origine landaise et 5 d'origine portugaise).

Pour chacun de ces couples landais portugais nous avons comparé les moyennes des teneurs des 8 éléments totaux dosés et les moyennes des trois notes: Note $E$, note globale novembre 1966 et note globale janvier-février 1967 par le test $t$ de FISHER et Yates (Tableau 6).

\section{TABleau 6}

Comparaison des moyennes des compositions foliaires et des notations de differents couples porrugais landais (Test t de Fisher et Yates) (* significatif au risque de $5 \%$ —* significatif au risque de $I \%$ )

\begin{tabular}{|c|c|c|c|c|}
\hline & Pissos & Lipostisey & Pallas & Dussillol \\
\hline $\mathrm{N} \ldots \ldots \ldots \ldots \ldots$ & 1,11 & 0,05 & $-3,69^{* *}$ & $-1,25$ \\
\hline $\mathrm{P}_{2} \mathrm{O}_{5} \quad \ldots \ldots \ldots \ldots \ldots$ & $2,90^{*}$ & 0 & 0,43 & $4,43^{* *}$ \\
\hline $\mathrm{K} \ldots \ldots \ldots \ldots \ldots$ & $-0,49$ & 0,90 & 0,93 & $3,06^{* *}$ \\
\hline $\mathrm{Ca}, \ldots . . . . . . . . .$. & 0,98 & 2,12 & 0,08 & 1,60 \\
\hline $\mathrm{Mg}_{\mathrm{g}} \ldots \ldots \ldots \ldots \ldots$ & 1,30 & -1.68 & 0,60 & 1,38 \\
\hline$M n \ldots \ldots \ldots \ldots \ldots$ & 0,90 & 1,87 & 0,61 & 1,70 \\
\hline $\mathrm{Cu} \quad \ldots \ldots \ldots \ldots \ldots$ & $3,33^{\circ}$ & $-0,59$ & 1,60 & 2,02 \\
\hline B $\ldots \ldots \ldots \ldots \ldots$ & 0,51 & 1,11 & 1,22 & 1,30 \\
\hline$E \ldots \ldots \ldots \ldots \ldots \ldots$ & $11,72^{* *}$ & $3,46^{* *}$ & $5,17 * *$ & $4,30^{* *}$ \\
\hline NG $66 \ldots \ldots \ldots \ldots$ & $7,30^{* *}$ & $6,04^{* *}$ & $4,30^{* *}$ & $7,30^{* *}$ \\
\hline NG $67 \ldots \ldots \ldots \ldots$ & $12,86^{* *}$ & $3,78 * *$ & $9,25 * *$ & $11,20^{* *}$ \\
\hline
\end{tabular}

Nous constatons pour ces quatre couples que les moyennes des trois notes diffèrent très significativement entre landais et portugais.

Pour deux de ces couples, la nutrition diffère significativement pour deux éléments, $\mathrm{P}_{2} \mathrm{O}_{5}$ et $\mathrm{Cu}$ dans le cas de Pissos, $\mathrm{P}_{2} \mathrm{O}_{5}$ et $\mathrm{K}$ dans le cas de Dussillol. 
Nous ne pouvons dans ces deux cas attribuer les différences de comportement uniquement à des différences d'origine.

Il faut cependant noter que dans le cas de Pissos la note $E$ diffère très significativement entre landais et portugais, alors que dans la nutrition aucun des éléments intervenant dans le déterminisme de la chute des aiguilles ne montre de différences significatives.

Pour les deux autres couples (Liposthey et Pallas), si les notations des deux origines diffèrent moins que pour Pissos et Dussillol, elles n'en sont pas moins très significativement différentes ; les notes les plus mauvaises étant attribuées à l'origine portugaise.

Cette fois, nous n'observons pas de différences dans la nutrition, sauf pour l'azote dans le cas de Pallas, mais au bénéfice des Pins portugais ( $t$ négatif).

Pour ces deux propriétés de Liposthey et de Pallas, notre hypothèse se trouve parfaitement vérifiée.

Dans les deux cas, nous observons des différences très significatives de comportement entre les peuplements d'origine supposée différente, sans qu'il y ait aucune différence significative dans la nutrition au profit des Pins d'origine landaise, alors que leur note est bien supérieure à celle des Pins d'origine portugaise.

La différence de comportement vis-à-vis du dépérissement en mauvaises conditions de nutrition minérale ne peut donc être attribuée qu'à l'origine portugaise (ou présumée telle) des graines; les Pins portugais s'accomodent beaucoup plus mal de déficiences alimentaires que les Pins d'origine locale.

\subsection{Jugement porté sur la nutrition}

\subsection{Jugement en valeur absolue}

Il est difficile de donner des valeurs optimales de composition foliaire valables pour toutes les essences résineuses et dans tous les cas.

Nous pouvons seulement donner un tableau des teneurs normales pour des peuplements en bon état, et des seuils de carence généralement admis, étant entendu que ces valeurs sont susceptibles de variations assez importantes au moins pour les valeurs optimales.

Si nous comparons les valeurs moyennes de composition foliaire des différents peuplements étudiés (tableau 4) aux valeurs optimales (tableau 7), nous constatons immédiatement, même en admettant que les valeurs du tableau 7 soient approximatives, que le niveau de nutrition des deux origines est faible.

Il est très faible pour les deux éléments majeurs, $\mathrm{N}, \mathrm{P}_{2} \mathrm{O}_{5}$, dont les teneurs moyennes descendent nettement au-dessous du seuil de carence et moins faible comparativement pour le potassium et les trois oligoéléments qui sont présents dans les aiguilles à des concentrations faibles mais le plus souvent supérieures au seuil de carence. L'intervention de ces quatre derniers éléments dans l'explication des phénomènes 


\section{TABLEAU 7}

Teneur normale et seuils de carence de 8 éléments dons les aiguilles de résineux en général

\begin{tabular}{l|c|c|c|c|c|c|c|c|c|}
\hline \hline & $\begin{array}{c}\mathrm{N} \\
\%\end{array}$ & $\begin{array}{c}\mathrm{P}_{2} \mathrm{O}_{5} \\
\%\end{array}$ & $\begin{array}{c}\mathrm{K} \\
\%\end{array}$ & $\begin{array}{c}\mathrm{Ca} \\
\%\end{array}$ & $\begin{array}{c}\mathrm{Mg} \\
\%\end{array}$ & $\begin{array}{c}\mathrm{Mn} \\
\%\end{array}$ & $\begin{array}{c}\mathrm{Cu} \\
\text { p.p.m. }\end{array}$ & $\begin{array}{c}\mathrm{B} \\
\text { p.p.m. }\end{array}$ \\
\hline Teneurs optimales & $1,8-2$ & $0,35-0,40$ & 0,50 & variable & 0,10 & variable & $6-8$ & $14-30$ \\
\hline Seuils de carence & 1,1 & 0,23 & $0,35-0,40$ & 0,03 & 0,05 & 0,03 & $4-5$ & 12 \\
\hline \hline
\end{tabular}

de dépérissement pose donc la question des teneurs optimales dans les aiguilles du Pin maritime d'origine portugaise.

\subsection{Possibilité d'amélioration de la nutrition}

Le dépérissement est donc caractérisé par un état nutritionnel très déficient. Nous pouvons donc espérer lutter contre le dépérissement en corrigeant cette déficience.

Il est intéressant à ce sujet de comparer les compositions foliaires des peuplements landais, dont on sait qu'ils réagissent à la fertilisation et les compositions foliaires de peuplements portugais en voie de dépérissement.

Nous pouvons par exemple prendre les placeaux témoins de l'expérience de Mimizan et deux peuplements dépérissants types Pallas et Liposthey (tableau 4).

Il n'y a guère de différence entre les deux peuplements portugais et le peuplement landais sauf pour l'azote dont la teneur à Mimizan tout en étant très faible $(1,14 \%)$ est cependant supérieure à celle de Pallas et Liposthey.

Or à Mimizan, l'effet de la fertilisation a été considérable. Nous pouvons donc, dans le cas de ces peuplements portugais qui ont une nutrition aussi ou plus déficiente espérer une amélioration de cette nutrition par fertilisation et par conséquent une amélioration de l'état des peuplements.

Nous aboutissons d'ailleurs à la même conclusion en comparant la nutrition des peuplements portugais en bon état et celle de peuplements atteints.

Deux exemples de peuplements portugais exempts de dépérissement peuvent être donnés par la propriété Rasson (ancienne zone cultivée) et la propriété Herré à Sourbé située sur sables fauves (tableau 4).

La nutrition, si elle n'atteint pas encore un niveau extraordinaire, est néanmoins bien meilleure que celle des peuplements atteints.

La teneur en azote dépasse $1 \%$, la teneur en phosphore atteint $0,30 \%$ et la teneur en potassium: $0,55 \%$.

\section{4. - DÉPÉRISSEMENT ET PROPRIÉTÉS CHIMIQUES DES SOLS}

Après avoir mis en évidence l'influence du type de sol sur l'intensité du dépérissement puis démontré par analyses foliaires que le déterminisme de ce dépérisse- 
ment était en partie d'ordre nutritionnel, il était nécessaire d'étudier les propriétés chimiques des sols en relation avec leur type génétique et en relation avec le dépérissement.

Nous avons vu par analyses foliaires le rôle fondamental des trois éléments $\mathrm{P}_{2} \mathrm{O}_{5}, \mathrm{~K}$ et $\mathrm{N}$ et à degré moindre celui de deux oligoéléments $\mathrm{Mn}$ et $\mathrm{Cu}$.

Dans l'étude du sol, nous nous sommes pour l'instant surtout attachés au phosphore et au potassium. Mais il est bien certain que l'étude du cuivre et du manganèse sommairement effectuée pour ce dernier élément, mériterait à l'avenir une étude plus importante.

De même pour l'azote, l'étude n'a pour l'instant été effectuée que par la détermination du rapport $\mathrm{C} / \mathrm{N}$. Un programme important sur le capital d'azote dans les sols et son taux de minéralisation serait indispensable.

L'étude des relations entre le phosphore et le potassium du sol et le dépérissement a été effectuée en 1965 en utilisant par conséquent l'ancienne notatıon visuelle (note s'échelonnant de 0 à VI).

Les résultats obtenus ayant été jugés satisfaisants, il ne nous a pas semblé nécessaire de les confirmer en utilisant la nouvelle notation.

Une difficulté s'est présentée immédiatement pour ce travail : la teneur en éléments minéraux est très variable d'un horizon à l'autre. Il était donc nécessaire d'effectuer les comparaisons horizon par horizon. Or le plus souvent ceux-ci ne sont pas comparables d'un type de sol à l'autre. Le seul horizon commun à tous les types de sols est l'horizon $A_{1}$. Nous n'avons donc comparé que celui-ci, ce qui avait d'ailleurs un autre avantage. En effet, les horizons supérieurs organiques sont beaucoup plus riches que les horizons sous-jacents en raison du jeu du cycle biologique. Les différences sont ainsi rendues beaucoup plus facilement décelables.

\section{1. - Influence de phosphore}

En raison de l'extrême pauvreté des sols landais, les dosages du phosphore sont extrêmement délicats car les méthodes usuelles sont à leur limite de sensibilité et il devient ainsi difficile de différencier des stations entre elles.

Nous avons tout d'abord utilisé la méthode habituelle de dosage du phosphore, dit assimilable, dans les sols forestiers : double extraction à l'acide sulfurique dilué et à la soude.

Par cette méthode, la teneur en $\mathrm{P}_{2} \mathrm{O}_{5}$ assimilable est de l'ordre de $0,04 \%$ et à peu près constante quel que soit le type de sol ou les stations, même là où par analyse foliaire il est évident que des différences notables existent.

De nouveaux dosages effectués en augmentant la prise d'essai n'ont guère donné de meilleurs résultats, la quantité de matière organique extraite par la soude n'autorisant que des mesures colorimétriques tout à fait approximatives. 
Nous nous sommes alors tournés vers un mode d'extraction plus puissant, l'acide chlorhydrique à $25 \%$ à chaud.

La silice a été éliminée par évaporation à sec et un traitement à l'acide perchlorique concentré. Ce traitement supprime également l'action perturbatrice du fer sur la colorimétrie en transformant les sels de fer en perchlorates incolores. La colorimétrie du phosphore a ensuite été effectuée par le vanadomolybdate.

Par cette méthode, nous obtenons des teneurs en $\mathrm{P}_{2} \mathrm{O}_{5}$ semi-total toujours très faibles variant de 0,06 à $0,10 \%$ dans la plupart des stations de la Grande Lande.

Des variations aussi faibles ne permettent guère de différencier les types de sols entre eux.

Par contre, si nous prenons les sols des peuplements portugais exempts de dépérissement et pour lesquels l'analyse foliaire donnait des teneurs en $\mathrm{P}_{2} \mathrm{O}_{5}$ dans les aiguilles supérieures à $0,30 \%$, la méthode d'extraction à l'acide chlorhydrique donne dans tous les cas des teneurs supérieures à $0,15 \%$ (Rasson ancien champ 0,17 à $0,27 \%$ - Lublon ancien champ $0,16 \%$, Murret ancienne bergerie $0,27 \%$, Herré sables fauves $0,21 \%$ ). Il est remarquable de constater qu'une teneur en $\mathrm{P}_{2} \mathrm{O}_{5}$ semi-total au moins égale à $0,15 \%$ oo dans l'horizon $\mathrm{A}_{1}$, est suffisante pour empêcher l'apparition du dépérissement quel que soit le type de sol et par conséquent le degré d'hydromorphie.

\section{Tableau 8}

Teneur du sol en $\mathrm{P}_{2} \mathrm{O}_{5}$ semi total et état du peuplement (ancienne notation)

\begin{tabular}{|c|c|c|c|c|c|}
\hline \multirow{2}{*}{ Propriétés } & \multirow{2}{*}{ Origine } & \multirow{2}{*}{ Peuplement } & \multicolumn{2}{|c|}{ Sol } & \multirow{2}{*}{$\begin{array}{c}\mathrm{P}_{2} \mathrm{O}_{5} \% \\
\mathrm{HCl} \text { à } 25 \% \\
\text { Horizon }\end{array}$} \\
\hline & & & Type & Conditions & \\
\hline Rasson ................ & $\mathbf{P}$ & VI & 6 & ancien champ & 0,17 \\
\hline Rasson $\ldots \ldots \ldots \ldots \ldots \ldots$ & $\mathbf{P}$ & VI & 4 & ancien champ & 0,17 \\
\hline Rasson $\ldots \ldots \ldots \ldots \ldots$ & $\mathrm{P}$ & VI & 3 & ancien champ & 0,17 \\
\hline Rasson $\ldots \ldots \ldots \ldots \ldots$ & L & VI & 1 & hors champ & 0,06 \\
\hline Rasson $\ldots \ldots \ldots \ldots \ldots$ & $\mathrm{P}$ & III-IV & $3-4$ & hors champ & 0,10 \\
\hline Rasson .................... & $\mathrm{P}$ & $0-1$ & 5 & hors champ & 0,10 \\
\hline Lublon .................. & $\mathrm{P}$ & VI & 1 & ancien champ & 0,16 \\
\hline Lublon .................. & $\mathrm{P}$ & $\mathrm{v}$ & 6 & ancien champ & 0,16 \\
\hline Murret $\ldots \ldots \ldots \ldots \ldots$ & $\mathbf{P}$ & VI & 3 & $\begin{array}{l}\text { ancienne } \\
\text { bergerie }\end{array}$ & 0,27 \\
\hline Sourbe ................. & $\mathbf{P}$ & II & sol brun acide & sables fauves & 0,21 \\
\hline
\end{tabular}




\section{2. - Influence du potassium}

Le potassium, autre élément fondamental après le phosphore, a été étudié en détail dans deux propriétés, la propriété Rasson (zone hors champ) et la propriété Péreire.

Dans ce cas, les analyses foliaires ont montré un rôle important du potassium.

\subsection{Propriété Rasson}

Nous avons tout d'abord essayé de déterminer s'il existait une liaison entre le dépérissement et la teneur en potassium échangeable du sol, teneur qui est partout faible, de l'ordre de 0,10 m.e. pour $100 \mathrm{~g}$. Nous n'avons trouvé aucune corrélation.

Par contre, nous avons pu montrer qu'il existait une liaison significative entre type de sol, donc hydromorphie et taux de saturation (fig. 5). La capacité totale

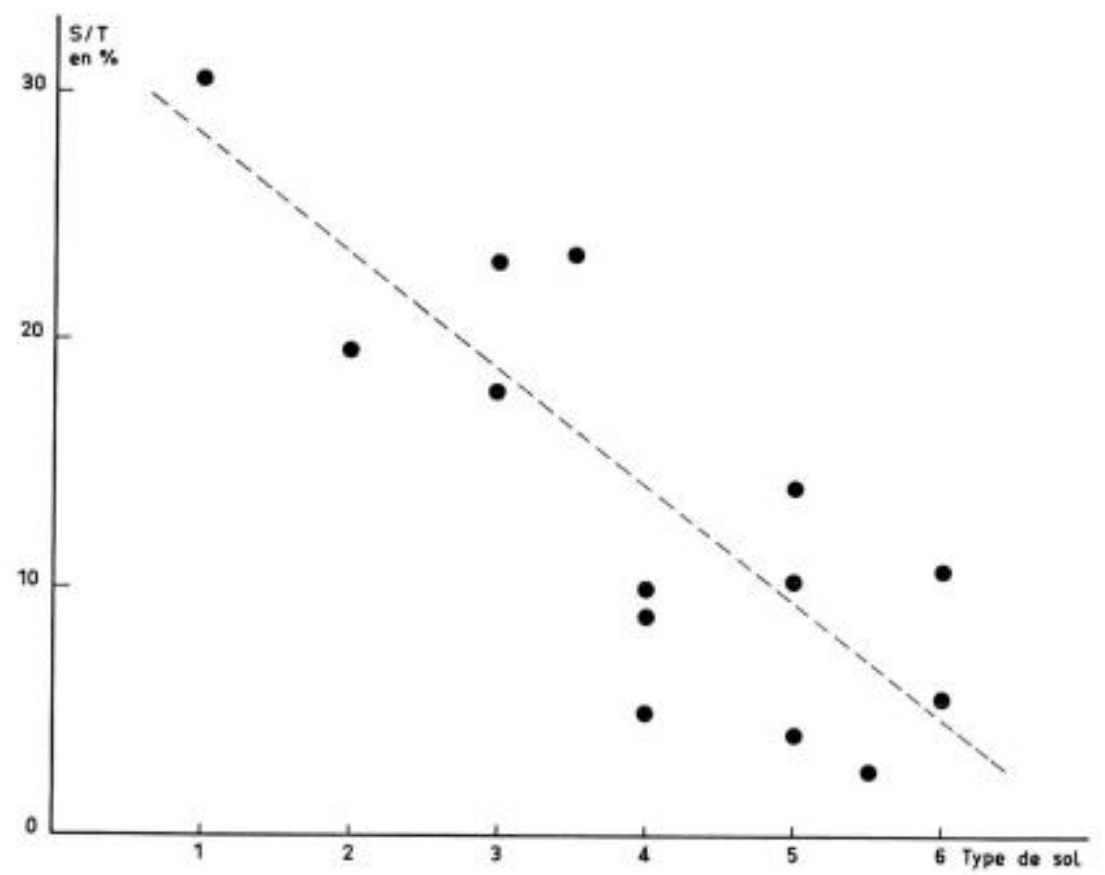

FıG. 5. - Corrélation entre taux de saturation et type de sol (propriété Rasson).

d'échange étant relativement constante, la variation du taux de saturation est due à la variation de la teneur en calcium et magnésium échangeables, la teneur en potassium échangeable restant constante. Plus les sols sont hydromorphes plus le taux de satu- 
ration est faible. Or, nous avons vu qu'il existait une liaison très significative entre hydromorphie et dépérissement.

Nous pouvons donc avancer l'hypothèse suivante : la teneur en potassium échangeable étant faible et assez peu variable, son absorption est surtout fonction du taux de saturation.

En sol peu influencé par l'hydromorphie, le taux de saturation étant élevé, l'absorption du potassium sera aisée. Par contre en sol hydromorphe, bien que la teneur en potassium échangeable soit équivalente, en raison de la très forte désaturation du complexe absorbant, son absorption sera difficile.

\subsection{Propriété Péreire}

Cette propriété située dans le département de la Gironde présente une variation continue d'est en ouest dans l'intensité du dépérissement.

Une cinquantaine d'observations sol-peuplement effectués suivant l'axe est-ouest ne nous a pas permis de mettre en évidence une relation entre le type de sol et l'intensité du dépérissement contrairement à ce que nous avions observé pour l'ensemble des peuplements atteints.

Cette propriété est la seule, avec une autre du département des Landes, où une telle corrélation n'ait pu être observée.

Les sols de cette propriété sont d'ailleurs assez homogènes et appartiennent surtout aux types 2 et 4 . Cette relative homogénéité explique en partie cette absence de corrélation confirmée par le test du $\chi^{2}\left(\chi^{2}\right.$ non significatif).

Il faut donc chercher ailleurs une explication possible. Nous pouvons supposer qu'un des deux éléments ou les deux éléments essentiels $\mathrm{P}_{2} \mathrm{O}_{5}$ et $\mathrm{K}$ varient d'est en ouest dans le même sens que le dépérissement ; cette variation pouvant dépendre de la composition minéralogique du sable.

Nous avons comparé les horizons $A_{1}$ de 16 sols répartis tout le long de l'axe est-ouest. La teneur en phosphore semi-total est à peu près constante, de l'ordre de $0,08 \%$, et done faible.

Nous avons donc essayé de déterminer le rôle éventuel du potassium tout d'abord en essayant de lier le dépérissement et le taux de saturation bien que l'hydromorphie soit peu variable.

Or, nous n'avons trouvé aucune relation entre dépérissement et taux de saturation, ce taux de saturation étant relativement peu variable et assez élevé dans l'ensemble.

Par contre, contrairement à ce que nous avions observé pour la propriété RAsson, la teneur en potassium échangeable est variable et le calcul du coefficient de corrélalation entre dépérissement et teneur en potassium échangeable du sol montre qu'il est très significatif. Il est de 0,76 pour un nombre de degrés de liberté de 15 ( $r$ table au risque de $5 \%=0,48$ ). Il existe donc une liaison hautement significative entre la quantité de potassium échangeable du sol et l'intensité du dépérissement (fig. 6). 


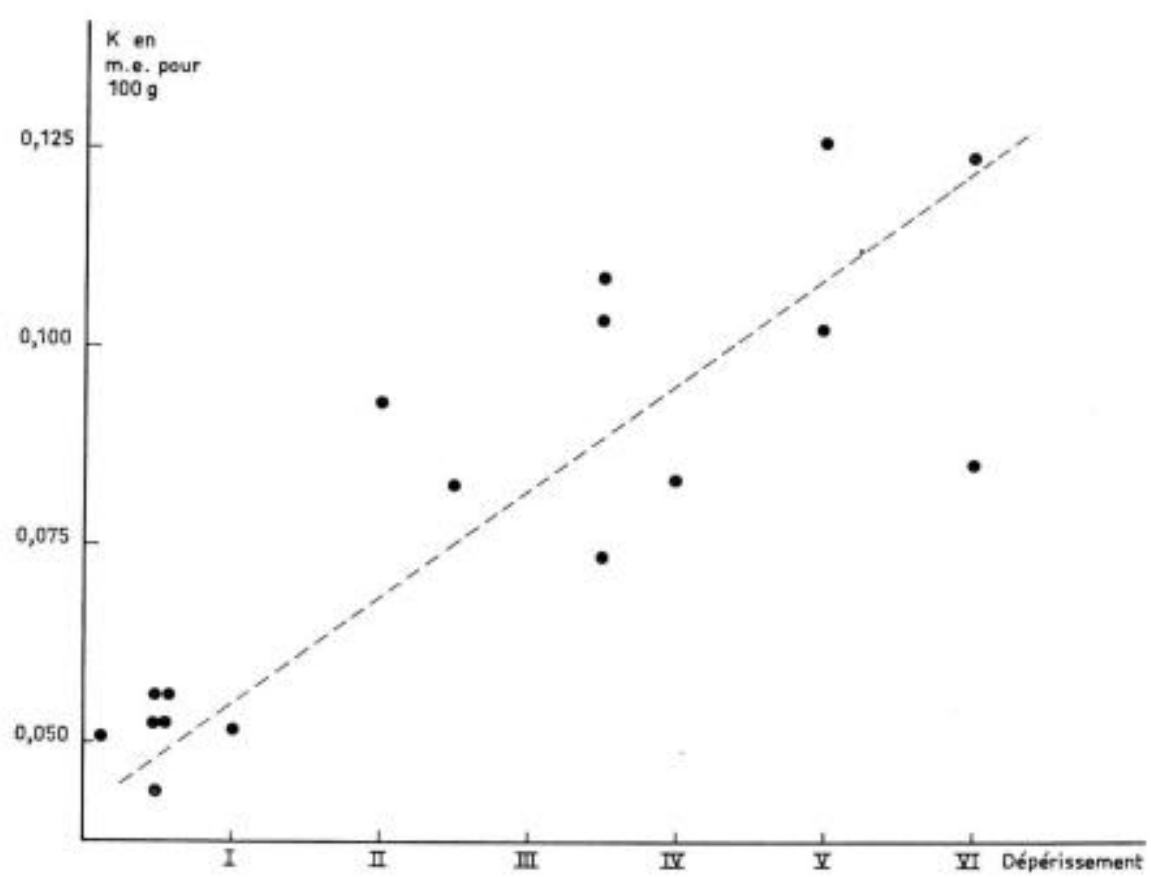

Fig. 6. - Corrélation entre teneur du sol en potassium échangeable (horizon AI) et dépérissement (Proprieté Pereire).

Le dosage du potassium extrait à $\mathrm{HCl} 25 \%$ bouillant, donc par la même méthode d'extraction qui s'était révélée intéressante pour l'acide phosphorique, n'a pas fourni de résultats plus valables que la détermination du potassium échangeable.

\section{3. - Infltrence de l'aluminium}

D'après les travaux de JUSTE, on sait que l'aluminium échangeable peut être toxique et que, de plus, il est abondant dans les horizons superficiels des sols hydromorphes. 11 est donc possible qu'il joue un rôle dans les désordres de nutrition qui ont été constatés et explique en partie la relation entre hydromorphie des sols et dépérissement. Cependant, nous n'avons pas étudié ce problème qui aurait nécessité une campagne supplémentaire importante sur le terrain et des expérimentations spéciales. Un simple dosage de l'aluminium échangeable dans les horizons superíciels des différents sols aurait très vraisemblablement montré une corrélation entre cet élément et le dépérissement, sans nous apporter une preuve certaine de son rôle. Les dosages d'aluminium libre (réactif de Tamm) que nous avons effectués ont d'ailleurs confirmé la plus grande abondance de cette forme dans les horizons supérieurs des sols hydromorphes (fig. 2). 
Dans le cas où le rôle de l'aluminium échangeable serait certain, la thérapeutique à envisager consisterait en un simple chaulage qui diminue le taux d'aluminium échangeable : or, on sait qu'un apport de calcaire n'a aucun effet sur les semis de Pin souffrant de carence en phosphore (P. MAUGE) dont la croissance n'est stimulée que par l'apport d'engrais phosphaté. Des recherches plus amples sur le rôle de l'aluminium, pour intéressantes qu'elles soient, nous semblaient donc n'avoir que peu de chances de déboucher sur un résultat pratique.

\section{4. - Conclusions}

L'étude de l'influence des propriétés chimiques du sol sur le dépérissement, effectuée surtout pour le potassium et le phosphore, confirme et complète les résultats obtenus par analyses foliaires bien que ces analyses de sols permettent une analyse beaucoup moins fine du phénomène.

Nous pouvons estimer que lorsque la teneur en $\mathrm{P}_{2} \mathrm{O}_{5}$ semi-total du sol est supérieure à $0,15 \%$, au moins dans les premiers $\mathrm{cm}$, le dépérissement ne se manifeste pas. Naturellement, la plupart des sols landais contiennent toujours des quantités inférieures. Dans le cas où le seuil de $0,15 \%$ n'est pas atteint et si la teneur en $\mathrm{P}_{2} \mathrm{O}_{5}$ est relativement constante et faible, il y a apparition du dépérissement dont I'intensité dépendra de la disponibilité d'un deuxième élément, le potassium.

Le plus souvent, cet élément est en quantité faible et inférieure à 0,10 m.e. pour $100 \mathrm{~g}$ (K échangeable).

Son absorption sera d'autant plus difficile que le complexe absorbant sera désaturé, la désaturation augmentant avec l'hydromorphie, ce qui est le cas général.

Si l'hydromorphie est peu variable et le taux de saturation constant, l'absorption du potassium sera directement fonction de la quantité de potassium échangeable du sol (propriété Péreire).

\section{5. - LES REMÉDES POSSIBLES}

\section{1. - Le drainage}

Nous avons vu que le dépérissement est intense surtout dans les zones les plus hydromorphes. Le tableau 9 correspondant à des exemples pris dans la propriété Rasson montre que la qualité du peuplement et sa nutrition se dégradent nettement lorsqu'on s'éloigne d'un fossé.

Le drainage est donc une des premières améliorations à apporter. Les reboiseurs landais le savent d'ailleurs bien et des efforts importants sont faits dans ce sens. Les exemples ci-dessus montrent cependant que l'effet des fossés n'excède pas 20 à $25 \mathrm{~m}$ et donc que la distance entre deux fossés devrait être au maximum de 40 à $50 \mathrm{~m}$. 


\section{TABLEAU 9}

Influence du drainage sur la nutrition

\begin{tabular}{c|c|c|c|c|c|c|c|c}
\hline \hline 1 & $\begin{array}{c}\text { Peuple- } \\
\text { ment }\end{array}$ & $\mathrm{N} \%$ & $\mathrm{P}_{2} \mathrm{O}_{5} \%$ & $\mathrm{~K} \%$ & $\mathrm{Ca} \%$ & $\mathrm{Mg} \%$ & $\mathrm{Mn} \%$ & $\begin{array}{c}\mathrm{Cu} \\
\text { p.p.m. }\end{array}$ \\
\hline $5 \mathrm{~m}$ du drain ... & III-IV & 1,00 & 0,27 & 0,40 & 0,25 & 0,14 & 0,06 & 2,8 \\
\hline $25 \mathrm{~m}$ du drain ... & III-IV & 0,94 & 0,23 & 0,46 & 0,36 & 0,13 & 0,05 & 2,1 \\
\hline $50 \mathrm{~m}$ du drain ... & I & 0,75 & 0,21 & 0,40 & 0,25 & 0,10 & 0,02 & 1,0 \\
\hline $2^{2}$ Série & & & & & & & & \\
\hline $5 \mathrm{~m}$ du drain ... & IV & 1,12 & 0,20 & 0,35 & 0,27 & 0,10 & 0,02 & 3,1 \\
\hline $30 \mathrm{~m}$ du drain ... & I-II & 1,09 & 0,17 & 0,30 & 0,16 & 0.14 & 0,02 & 2,4 \\
\hline \hline
\end{tabular}

\section{2. - La fertilisation}

Dès que furent connus les résultats des premières analyses foliaires, la décision fut prise d'installer dans les peuplements dépérissants des essais de fertilisation. L'un même, celui de Sore, fut installé dès 1964 dans un reboisement en très mauvais état avant toute étude systématique des causes du dépérissement.

Nous donnons, ci-dessous, pour chacun de ces essais, une description rapide des principaux traitements, tandis que les tableaux 10 et 11 en donnent les résultats.

\subsection{Essai de Sore (Landes)}

Il a été effectué pendant l'hiver 1964-1965 dans un peuplement très fortement atteint, sur lande sèche à callune avec un peu de molinie et avec des creux à Erica ciliaris et Erica tetralix. On a cherché à tester à la fois le travail du sol qui a consisté en un passage de rotavator suivi d'un labour entre les bandes de pins et la fertilisation qui a apporté $\mathrm{N}, \mathrm{P}$ et $\mathrm{K}$, seuls ou en combinaison suivant les formules suivantes :

$\mathrm{P}$ : $250 \mathrm{~kg}$ de $\mathrm{P}_{2} \mathrm{O}_{5}$ par ha labouré (soit environ $125 \mathrm{~kg}$ par ha cadastral), sous forme de scories à $18 \%$ enfouies par le labour en octobre 1964 ou simplement épandues en surface suivant les cas.

$\mathrm{K}: 250 \mathrm{~kg}$ de $\mathrm{K}_{2} \mathrm{O}$ par ha labouré, sous forme de sulfate de potasse à $50 \%$.

$\mathrm{N}: 80 \mathrm{~kg}$ par ha cadastral, sous forme d'ammonitrate à $34,5 \%$ épandu en surface en février 1965. Cet apport a été renouvelé en mai 1966.

Les traitements appliqués furent effectivement les suivants :

$\mathrm{T}_{0} \mathrm{E}_{0}$ : ni travail du sol, ni fertilisation,

$\mathrm{T}_{0} \mathrm{E}_{1}: \mathrm{P}, \mathrm{K}$ et $\mathrm{N}$ épandus en surface sans travail du sol,

$\mathrm{T}_{1} \mathrm{E}_{1}$ : travail du sol, fertilisation N P K ( $\mathrm{P} \mathrm{K}$ enfouis au labour, $\mathrm{N}$ en surface);

$T_{1} E_{2}$ : travail du sol et fertilisation $P$ enfouie au labour ;

$\mathrm{T}_{1} \mathrm{E}_{3}$ : travail du sol et fertilisation $\mathrm{N} \mathrm{K}$ ( $\mathrm{K}$ enfoui au labour et $\mathrm{N}$ en surface). 
Cet essai ne comporte pas de répétition mais constitue un des blocs d'un essai plus vaste, comprenant au total 3 blocs. L'ensemble de l'essai donnant des résultats significatifs et ces résultats étant pratiquement les mêmes dans les trois blocs, nous pouvons considérer que les différences constatées à Sore sont bien dues au traitement (tableau 10).

L'accroissement en hauteur mais aussi l'aspect général de vigueur du peuplement donné par la masse foliaire et la longueur des aiguilles ont été spectaculairement améliorés. Seul le traitement $T_{1} E_{1}$ est nettement efficace ; $T_{1} E_{2}$ l'est faiblement et les autres pratiquement pas. Cela montre donc que le travail du sol, l'apport de phosphore et d'azote sont indispensables pour obtenir un résultat valable. La suppression d'un seul de ces trois éléments du traitement conduit à un effet notablement plus faible ou nul.

TABLIAU 10

Essai de fertilisation de Sore

(* significatif aे $5 \%$ - ** significatif à $1 \%$ )

\begin{tabular}{|c|c|c|c|c|c|c|}
\hline \multirow{2}{*}{ Traitement } & \multicolumn{2}{|c|}{ Sans labour } & \multicolumn{4}{|c|}{ Avec labour } \\
\hline & $\begin{array}{c}\text { Non fertil. } \\
T_{0} E_{0}\end{array}$ & $\begin{array}{l}\mathrm{NPK} \\
\mathrm{T}_{0} \mathrm{E}_{1}\end{array}$ & $\begin{array}{l}\text { Non fertil. } \\
\mathrm{T}_{1} \mathrm{E}_{0}\end{array}$ & $\begin{array}{l}N P K \\
T_{1} E_{r}\end{array}$ & $\underset{T_{1} E_{2}}{P}$ & $\begin{array}{l}\text { NK } \\
T_{1}: 3\end{array}$ \\
\hline $\begin{array}{l}\text { Accroissement en hau- } \\
\text { teur en } \mathrm{cm}(66 \text { et } 67)\end{array}$ & 36 & 19 & 33 & $69^{* *}$ & $52^{4}$ & 36 \\
\hline
\end{tabular}

\subsection{Essai de la propriété Rasson à Retjons (Landes)}

Il a été installé en 1965. Rappelons qu'il s'agit d'un peuplement âgé de 11 ans en 1966, en lande très humide à molinie ; on a combiné en plusieurs types de traitement les interventions suivantes :

Travail du sol : labour entre les bandes de pins en avril 1965.

Fertilisation $P: 250 \mathrm{~kg}$ de $\mathrm{P}_{2} \mathrm{O}_{5}$ par ha d'interbande (125 kg par ha cadastral) sous formes de scories enfouies par labour.

Fertilisation $K: 250 \mathrm{~kg}$ de $\mathrm{K}_{2} \mathrm{O}$ par ha d'interbande sous forme de sulfate de potassz à $50 \%$ enfoui au labour.

Fertilisation $N: 50 \mathrm{~kg}$ d'azote par ha cadastral appliqué en surface au printemps 1965, puis $80 \mathrm{~kg}$ en 1966 sous forme d'ammonitrate à $34,5 \%$.

Fertilisation N P K en surface : $125 \mathrm{~kg}$ de $\mathrm{P}_{2} \mathrm{O}_{5}, 125 \mathrm{~kg}$ de $\mathrm{K}_{2} \mathrm{O}$ et $62 \mathrm{~kg}$ d'azote par ha cadastral appliqués en surface au printemps 1965 sous forme d'engrais complet 8-16-16, complétée en 1966 par $80 \mathrm{~kg}$ d'azote de l'ammonitrate à $34,5 \%$.

Fertilisation $\mathrm{Mn}$ : $10 \mathrm{~kg}$ de $\mathrm{Mn}$ par ha d'interbande en avril 1965 sous forme de sulfate de Mn, soit enfoui par le labour, soit en surface, puis $15 \mathrm{~kg}$ de $\mathrm{Mn}$ apporté en surface en juillet 1965 . 
Ces interventions ont été combinées suivant les traitements suivants :

00 : ni labour, ni fertilisation

01 : pas de labour. Fertilisation N P K Mn en surface

02 : pas de labour. Fertilisation N P K en surface

10 : labour. Pas de fertilisation

11 : labour. Fertilisation P K enfouie. Fertilisation $\mathrm{N}$ et $\mathrm{Mn}$

12 : labour. Fertilisation P K enfouie. Fertilisation N

13 : labour. Fertilisation P K enfouie

14 : labour. Fertilisation N K (K enfoui)

15 : labour. Fertilisation N P (P enfoui).

Le tableau 11 donne les accroissements des années 1966 et 1967 en hauteur, circonférence et « volume » (en réalité, il ne s'agit que du produit $C^{2} h / 4 \pi$, c'est-à-dire le volume divisé par le coefficient de forme, qu'on suppose égal dans les différents traitements). Si nous considérons seulement cet accroissement en " volume n nous voyons que tous les traitements sont significatifs à $1 \%$ par rapport au témoin, sauf le travail sans fertilisation (10) qui n'est significatif qu'à $5 \%$ et a un effet faible. Cependant, les trois traitements 11,12 et 15 (N P K Mn, N P K, et N P) sont significativement supérieurs à $1 \%$ à tous les autres sauf au traitement sans travail du sol 01 (NPKMn en surface). Nous pouvons en conclure que les apports de phosphore et d'azote sont indispensables. De même, les comparaisons entre les traitements 10 et 00,11 et 01,12 et 02 nous montrent que le travail du sol est très utile. Par contre, les comparaisons entre les traitements 01 et 02 d'une part, 11 et 12 d'autre part ne permettent de mettre en évidence aucun effet certain du manganèse.

Sur le plan pratique, le traitement associant travail du sol, fertilisation azotée et fertilisation phosphatée parait le plus indiqué. Notons cependant qu'une fertilisation en surface apportant les quatre éléments N, P, K et Mn mais qui économise le labour ne lui est pas significativement inférieure. L'une et l'autre de ces méthodes permettent de doubler l'accroissement en volume deux années après l'application des engrais et en même temps de redonner au peuplement un aspect sain et vigourcux en augmentant considérablement la masse foliaire : les aiguilles sont plus longues, tous les verticilles de l'arbre sont feuillés alors que dans les témoins les deux ou trois verticilles inférieurs ont perdu toutes leurs aiguilles. Une détermination de la note $E$ effectuée en 1968 dans trois traitements montre un effet des engrais parallèle à celui qui est constaté sur l'accroissement en volume (tableau 11). Faisons simplement deux remarques à propos de cette note $E$.

a) Elle est nettement inférieure à celle attribuée en janvier 1967. L'explication tient au fait déjà signalé ci-dessus que le projet de récolte de gemme avait conduit à utiliser en 1967 des pins supérieurs à la moyenne, tandis que la note de février 1968 , établie à partir d'un grand nombre représente exactement la moyenne du peuplement.

b) Cette amélioration de la note $E$ par l'engrais donne une idée encore sous-estimée de l'accroissement de vigueur et de masse foliaire des peuplements qui est véritablement très spectaculaire ; il aurait fallu, comme nous l'avons déjà dit ci-dessus, inclure dans la note $E$ la longueur moyenne des aiguilles. 
En ce qui concerne l'action du phosphore, on doit remarquer dans cet essai, comme dans le précédent, que le phosphore a été apporté sous forme de scories. On peut donc penser qu'il s'agit à la fois d'un effet du calcium apporté par cet engrais et du phosphore.

\section{TABleau 11}

Essai de ferrilisation de la propriétẻ Rasson (* significatif a $5 \%, *$ significatif à $1 \%$ )

\begin{tabular}{|c|c|c|c|c|c|c|c|c|c|c|c|}
\hline \multirow[b]{2}{*}{ Traitements } & \multicolumn{3}{|c|}{ Sans labour } & \multicolumn{6}{|c|}{ Avec labour } & \multicolumn{2}{|c|}{ p.p.d.s. } \\
\hline & $\begin{array}{c}\text { Non } \\
\text { fertil. } \\
00\end{array}$ & $\begin{array}{c}\text { NPK } \\
02\end{array}$ & $\begin{array}{l}\text { NPK- } \\
\text { Mn } \\
01\end{array}$ & $\begin{array}{c}\text { Non } \\
\text { fertil. } \\
10\end{array}$ & $\begin{array}{l}\text { NK } \\
14\end{array}$ & $\begin{array}{l}\text { PK } \\
13\end{array}$ & $\begin{array}{l}\text { NP } \\
15\end{array}$ & $\begin{array}{c}\text { NPK } \\
12\end{array}$ & $\begin{array}{l}\text { NPK- } \\
\text { Mn } \\
11\end{array}$ & $5 \%$ & $1 \stackrel{a}{a}$ \\
\hline $\begin{array}{cc}\begin{array}{c}\text { Accroissement } \\
\text { hauteur }\end{array} 66 & \text { en } \\
67 \mathrm{en} \mathrm{cm} & \text { et...... }\end{array}$ & 108 & 121 & 141 & 111 & 123 & 123 & 137 & 140 & 140 & 21,3 & 29,4 \\
\hline $\begin{array}{r}\text { Accroissement en } \\
\text { circonférence } 66 \\
\text { et } 67 \mathrm{~cm} \ldots . . .\end{array}$ & 4,1 & $\begin{array}{l}6,4 \\
\leftrightarrow \rightarrow 4\end{array}$ & 7,3 & 6,1 & 6,2 & $\begin{array}{l}6,2 \\
* *\end{array}$ & $\begin{array}{l}8,4 \\
* *\end{array}$ & $\begin{array}{l}8,4 \\
* *\end{array}$ & $\begin{array}{l}8.9 \\
* *\end{array}$ & 1,5 & 2,1 \\
\hline $\begin{array}{r}\text { Accroissement en } \\
\text { volume } 66 \text { et } 67 \\
\operatorname{dm}^{3} \ldots \ldots \ldots . .\end{array}$ & 12,8 & $\begin{array}{c}19,4 \\
* * *\end{array}$ & $\underset{* *}{23,1}$ & 16,9 & $\underset{* *}{18.3}$ & $\begin{array}{c}19,2 \\
* *\end{array}$ & $\underset{* *}{25,6}$ & $\begin{array}{c}25,4 \\
* * *\end{array}$ & 27,4 & 3,95 & 5,44 \\
\hline Note $E, \ldots \ldots \ldots$ & 3,43 & & & & & 4,20 & & 4,98 & & & 0,07 \\
\hline
\end{tabular}

Sur le plan théorique, il aurait été plus judicieux, pour tester l'effet de $\mathrm{P}_{2} \mathrm{O}_{5}$, d'employer du superphosphate triple (pauvre en Ca et sans soufre). Mais comme il s'agissait de chercher un remède pratique au dépérissement dans le minimum de temps, nous avons préféré utiliser un engrais phosphocalcique qui, corrigeant partiellement l'acidité du sol, avait le maximum de chances d'être efficace (il apporte d'ailleurs en plus quelques oligoéléments).

Nous pensons cependant que le phosphore a eu le rôle principal ; on sait que dans la plupart des sols landais, il est indispensable pour la croissance des semis et que, dans ce cas, le superphosphate, les scories, les phosphates naturels ont une action équivalente. De plus, il a été prouvé par P. MAUGÉ dans l'essai de Sabres que le calcium seul n'avait aucun effet sur la croissance du Pin, alors que l'ensemble P $+\mathrm{Ca}$ avait un effet hautement positif. Des analyses foliaires pratiquées à la fin de 1965 sur les pins fertilisés du contrat Rasson ont montré un accroissement important de la teneur en $\mathrm{P}_{2} \mathrm{O}_{5}$ des aiguilles $(0,30$ à $0,36 \%$ contre 0,15 à $0,18 \%$ pour les témoins).

Notons cependant qu'un effet favorable du calcium n'est pas exclu. La faible action du traitement 02 (N P K en surface) où l'on a utilisé un engrais complet moins riche en $\mathrm{Ca}$ que les scories, pourrait d'ailleurs le faire penser, encore que le maintien 
d'une concurrence herbacée active suffise à expliquer que la fertilisation ait agi moins fortement dans ce cas que lorsqu'elle est combinée avec le travail du sol. Dans l'essai de Sore, l'application de scories en surface sans labour n'a d'ailleurs eu non plus aucun effet, malgré la présence simultanée de phosphore et de calcium.

Nous pensons donc, en définitive, que nous sommes bien devant un effet du phosphore. D'ailleurs, un nouvel essai qui sera mis en place au cours de 1968 comparera l'effet des scories et celui du superphosphate triple.

\subsection{Essais de la propriété Péreire (Gironde)}

Nous ne ferons que les citer car ils sont plus récents que les précédents (été 1966) et la fertilisation n'a pû agir que pendant un an. Il a été mis en place un essai du type factoriel $2^{3}$ destiné à tester l'effet des trois éléments $\mathrm{N}, \mathrm{P}$ et $\mathrm{K}$ et dans lequel, à la fin de 1967, aucun effet de la fertilisation n'était visible. Par contre, un essai portant sur les doses commence à marquer et confirme que l'effet du phosphore seul est faible comparé à celui d'une fertilisation complète N P K.

Logiquement, le traitement NPK de l'essai factoriel aurait dû être efficace. Toutefois, l'essai est trop jeune pour que ces résultats méritent d'être pris en considération.

\subsection{Discussion des résultats}

Nous n'avons pour l'instant que quelques résultats d'essais de fertilisation relativement simples où ne sont testées qu'un certain nombre de modalités.

Ces premiers résultats d'amélioration de la production et de l'état général des arbres par fertilisation confirment les conclusions auxquelles nous avions abouti après les études de nutrition.

Il existe cependant quelques contradictions entre certains des résultats des essais et certaines conclusions de l'étude des facteurs explicatifs du dépérissement ; par exemple, l'interprétation statistique des analyses foliaires mettait en lumière un rôle très important du potassium dans l'état sanitaire et la croissance des peuplements : aussi bien pour la note globale que pour la croissance en hauteur et diamètre, la longueur des aiguilles ou la note $E$, il apparaissait comme l'un des éléments déterminants, qu'on en juge d'après les coefficients de corrélation totale ou d'après l'analyse de régression progressive. Or, son rôle en fertilisation est très faible. 11 en est de même pour le manganèse.

Les résultats obtenus dans les essais de fertilisation s'accordent mieux, en définitive, avec les conclusions tirées d'une interprétation banale des analyses foliaires, par simple comparaison avec les normes généralement admises pour les résineux, qu'avec les résultats d'analyses statistiques plus complexes.

Il est possible qu'une analyse préalable des composantes principales et l'analyse de régression multiple sur ces composantes principales aient été plus judicieuses. Il faut aussi faire remarquer que les analyses statistiques ont été effectuées sur une 
population d'arbres qui présentaient tous, même les plus vigoureux, des déficiences très nettes en $\mathrm{N}$ et $\mathrm{P}$. Or, dans les essais de fertilisation, $\mathrm{K}$ et $\mathrm{Mn}$ n'ont jamais été testés seuls, mais toujours sur des peuplements fertilisés en même temps en $\mathrm{N}$ ou $\mathrm{P}_{2} \mathrm{O}_{5}$ (ou $\mathrm{N}$ et $\mathrm{P}_{2} \mathrm{O}_{5}$ ) c'est-à-dire en fait, sur une population bien alimentée en l'un ou l'autre de ces éléments, donc différente de celle sur laquelle avaient porté les calculs.

\section{CONCLUSION GÉNÉRALE}

Au terme de ces études, nous pouvons avancer de manière quasi certaine que le dépérissement des jeunes reboisements de Pins maritimes dans les Landes résulte de la conjugaison de deux causes :

- une cause d’ordre génétique,

- une cause d'ordre nutritionel et ceci d'autant plus que d'après Delatour le Collisiopsis pinea ne paraît pas jouer un rôle déterminant.

Des années particulièrement froides ont joué sans doute un rôle important dans l'extériorisation des symptômes.

Les peuplements de race locale ne présentent aucun symptôme de dépérissement quelles que soient les conditions de sols. Par contre, pour les peuplements d'origine portugaise, il existe une liaison très significative entre intensité du dépérissement et intensité de l'hydromorphie en sols pauvres chimiquement.

Si la richesse du sol est suffisante, le dépérissement n'apparaît pas, même en station très humide. Il n'y a pas de signes de dépérissement si la teneur du sol en $\mathrm{P}_{2} \mathrm{O}_{5}$ semi-total est supérieure à $0,15 \%$. Si cette teneur n'est pas atteinte, l'intensité du dépérissement est fonction essentiellement de la disponibilité du potassium qui dépend elle-même, soit de la teneur en potassium échangeable du sol, soit du taux de saturation du complexe absorbant. Des études de nutrition effectuées par analyses foliaires parallèlement aux études de sols montrent d'une manière générale que la nutrition des peuplements dépérissants présente une grave insuffisance en phosphore et azote, moindre en potassium et certains oligoéléments.

La comparaison de la nutrition des deux origines en conditions de sols pauvres ne montre aucune différence significative alors que les différences de comportement sont très grandes (absence de dépérissement pour l'origine landaise).

Il apparait par diverses analyses statistiques que les variations de croissance en hauteur et en diamètre de ces peuplements dépérissants sont en liaison surtout avec l'alimentation en phosphore et en oligoéléments, cuivre, manganèse et bore, ces trois oligoéléments étant seuls significatifs en fin de régression.

La vigueur des arbres que nous avons rapportée à la longueur des aiguilles et à la durée de leur persistance est encore en corrélation avec la teneur des aiguilles en phosphore, mais en fin de régression $\mathrm{N}, \mathrm{K}$ et Ca seuls restent significatifs alors que les oligoéléments n'apparaissent plus. 
Plusieurs essais de fertilisation effectués depuis 1964 corroborent partiellement ces résultats. Des améliorations très nettes, parfois même spectaculaires, ont pû être obtenues en conjuguant un travail du sol et une fertilisation à base de scories et d'engrais azotés ( $125 \mathrm{~kg}$ de $\mathrm{P}_{2} \mathrm{O}_{5}$ et $130 \mathrm{~kg}$ d'azote par ha). L'accroissement en volume double rapidement et les arbres dont l'aspect dénudé à l'origine donnait les plus vives inquiétudes se couvrent d'aiguilles longues et abondantes.

Quelques contradictions apparaissent entre les résultats de l'étude des facteurs expliquant les variations observées sur le terrain et les résultats des premiers essais de fertilisation.

Le meilleur moyen de déterminer les facteurs de correction en fertilisation semble le jugement en valeur absolue de la nutrition.

Un certain nombre de questions reste à préciser : le rôle éventuel en fertilisation des oligoéléments n'a pas encore été totalement expérimenté ; les doses de phosphore et d'azote et les équilibres optima demandent encore à être précisés ; l'absence d'effet d'une fertilisation apportée en surface sans travail du sol ne peut être tenue pour acquise après deux ans seulement d'expérimentation ; la durée d'effet de la fertilisation pratiquée est inconnue.

Pourtant nous pensons pouvoir dire que dans ses grandes lignes le dépérissement du Pin maritime d'origine portugaise est convenablement expliqué et qu'un remède relativement simple et très efficace peut être proposé sous forme d'une fertilisation azotée et phosphatée, accompagnée d'un travail du sol. C'est une opération assez coûteuse (450 à $500 \mathrm{~F} / \mathrm{ha}$ ), d'un prix équivalent à celui d'un reboisement nouveau. Il n'est pas impossible cependant qu'elle soit avantageuse si elle s'applique à des peuplements dépérissants de 10 à 15 ans. Pour ceux-ci, il faudrait en effet ajouter au coût du reboisement celui de l'élimination du peuplement ancien qui n'est pas encore commercialisable. En outre, grâce à l'avance déjà prise par le peuplement ancien, même dépérissant, et à l'augmentation rapide de sa production après fertilisation, les sommes investies pour la restauration seraient vraisemblablement récupérables à plus court terme que celles dépensées pour la constitution d'un boisement nouveau.

Reçu pour publication en août 1968

\section{REMERCIEMENTS}

Que soient remerciés ici tous les membres du Service forestier des Landes de Gascogne qui nous ont aidé dans le choix des peuplements et dans l'installation des expériences, en particulier, MM. Dupont, Castaing et Arastia. C.N.R.F.

Nous remercions également M. MuLıer et les Techniciens de la Station de Biométrie du 


\section{SUMMARY \\ INFLUENCE OF THE CONDITIONS OF MINERAL NUTRITION IN THE WITHERING OF THE MARITIME PINE-TREE IN THE LANDES OF GASCOGNE}

The withering of young reafforestations of maritime pine-trees in the Landes of Gascogne results from two conjugated causes :

- The use of seeds of portuguese origin,

- an important shortage of nutrition in phosphorus and nitrogen, and, in a smaller degree, in potassium, manganese, copper and boron.

The withering appears only on pine trees of portuguese origin and the more hydromorphic the soils are, the more important it reveals itself. In rich soils, particulary in phosphorus, the symptoms of withering do not appear, even in very damp station.

In hungry soils, the pine trees coming from the Landes remain sound through the amount of nitrogen and of phosphorus of their needles is as low as the amount in pine trees of portuguese origin.

A nitrogenous and phosphated fertilization, together with an action on the soil permit, after two years, to renew the strength of the decayed trees, increasing considerably the length of their needles and the time of their duration ; the current increment is at the same time doubled. A mere fertilization in surface seems to produce only a far less important action.

\section{ZUSAMMENFASSUNG}

\section{Der Einfluss der Mineralstoffernährung auf das Absterben der Seestrandkiefer (Pinus pinaster Ait.) in den \& Landes de Gascogne $"$}

Das Absterben junger Bestānde der Seestrandkiefer ist auf das Zusammenwirken zweier Ursachen zurückzuführen :

- die Verwendung von Saatgut portugiesischer Herkunft,

- eine völlig unzureichende Stickstoff - und Phosphorernãhrung und eine mangelhafte Kali -, Kupfer - und Borernährung.

Das Absterben zeigt sich nur bei Seestrandkiefern portugiesischer Herkunft und ist um so bedeutender je vernässter die Böden. Auf năhrstoffreichen Böden, insbesondere an Phosphor, treten auch auf sehr nassen Bōden keine Absterbesymptome auf. Die Herkünfte aus den « Landes » bleiben auch auf nāhrstoffarmen Standorten gesund, obwohl der Stickstoff- und Phosphorgehalt ihrer Nadeln ebenso gering ist als jener der portugiesischen Herkünfte.

Durch eine Phosphor- und Stickstoffdüngung verbunden mit einer Bodenbearbeitung wird innerhalb von zwei Jahren die volle Wüchsigkeit der erkrankten Bäume wiederum erreicht; gleichzeitig ist damit eine bedeutende Zunahme der Nadellänge und des Nadelalters verbunden und der laufende Massenzuwachs verdoppelt sich. Eine alleinige Oberflächendüngung Scheint eine wesentlich schwächere Wirkung zu haben.

\section{RÉFÉRENCES BIBLIOGRAPHIQUES}

AFOCEL. Essais de culture et de fumure sur pin maritime.

BONNEAU M., 1962. Acquisitions actuelles et problèmes à résoudre dans le domaine de la fertilisation forestière. Rev, forest. fr., 3, mars 1962.

BONNEAU M., LE TACON F., 1966. Le rôle du facteur sol dans le dépérissement du pin maritime dans les landes de Gascogne. Premiers résultats. Rapport ronéotypé.

BoUvarel P., 1960. Note sur la résistance au froid de quelques provenances de Pin maritime. Rev. forest. fr., 7, 495-508. 
Callek̀re S., Henin S., 1951. Etude des Alios des Landes. Ann. agron., 296-316.

Castaing P., 1957. Notice sur les principales méthodes de boisement (ou de reboisement) et d'équipement, appliquées aux Landes de Gascogne.

David-Lapraz G., 1960. Etude comparée de la croissance des pins des dunes et de landes. PV Soc. Sci. phys. nat. Bordeaux, séance du 5 mai 1960.

Delatour C., 1967. Contribution à l'étude de Caliciopsis pinea Peck : résultats d'inoculations artificielles et d'essais in vitro. Ann. Sci. forest., 24 (2), 157-175.

Delmas J., 1955. Landes de Gascogne, terre de sables. Agriculture, 164, 1-4.

Demounem R., 1956. Relations entre les sols, la composition floristique de la lande girondine et la croissance du Pin maritime, C.R. Acad. Sci., 261, 13, 2513-2516.

Duchaufour Ph., 1948. Note sur les sols landais et sur le problème de leur amelioration. C.R. Acad. Agric., 74.

Duchaufour Ph., 1949. L'évolution pédologique des sols landais. Cah. Ing. agron., 4, 41-43.

Duchaufour Ph., 1965. Précis de Pédologie, $2^{*}$ édition. Editions Masson. Paris.

Dupurs J., 1958. Observations pédologiques dans le Sud-Ouest de la France. Ann. Inst, nation. agron., XLIV, p. 60.

DUTIL. P., Juste C., 1964. Phytotoxicité de l'aluminium dans les sols des Landes, en relation avec la présence d'alios à faible profondeur. C.R. Acad. Agric., 50 (5), 4-11.

Enjalbert H., 1960. Les pays aquitains, le modèle et les sols, p. 607. Imprimerie Bière, Bordeaux.

Guinaudeau J., 1964. La Forêt landaise. INRA. Station de Recherches forestières de Bordeaux.

Guinaudeau J., Itley G., Mauge J.P., Dumas F., 1963. Essai de fertilisation minérale sur Pin maritime à Mimizan (Landes). Ann. Ecole, nation. Eaux Foréts, 20 (1).

Guinier Ph., 1945. La mise en valeur des landes de Gascogne et la création de la forêt landaise. L'ouvre de Chambrelent. Rev. Eaux Foréts, 83, 445-469.

Jacquin F., Juste C., Dureau (Mme P.), 1965. Contribution à l'étude de la matière organique des sols sableux des Landes de Gascogne. C. R. Acad. Agric., 8 décembre 1965, 1190-1197.

Juste C., DutIL P., 1965. Importance relative du fer et de l'aluminium dans les sols sableux des Landes de Gascogne, Sci, Sol, 1, 33-42.

Justz C., 1965. Contribution à Tétude de la dynamique de l'aluminium dans les sols acides du SudOuest atlantique : application à leur mise en valeur. Thèse Faculté des Sciences de Nancy, décembre 1965.

Kuhnholtz-Lordat, Barky J.P., Bernaux P., Bouchet Ch,, Renaud P., 1962. Evolution de la végétation des Landes de Gascogne d'après les amplitudes biologiques de quelques espèces à pouvoir dynamique élevé. Ann. agron., 3, 5 .

LALlemand P., 1951. Etude élémentaire agrologique de la région landaise. Bull. techn. agric., p. 14.

Lallemand P., Siloret et Sargos, 1953. La mise en valeur des Landes et l'économie agro-sylvopastorale. Congr, nation, Bois, Session Sud-Ouest, $\mathrm{n}^{\circ} 3$.

Lallemand P., Léonard J.P., Martin J., 1960. La sylviculture moderne du Pin maritime dans les Landes de Gascogne. Bull. techn. Inform. Ing. Serv, agric,, n' 150, mai 1960.

Nebe W., 1963. Uber die Beurteilung der Düngebedürftigkeit von Mittelgebirgs Standorten durch Blattenalysen. Arch. Forstw, 12 (10).

Seillac P., 1960. Contribution à l'étude de la nutrition du Pin maritime : variations saisonnières de la teneur des pseudophylles en azote, potassium et acide phosphorique. Thẻse Faculté des Sciences de Bordeaux.

TAmM C.O., 1964. Die Blattanalyse als Methode zur Ermittlung der Nährstoffversorgung des Waldes. Eine kritische Betrachtung. Tagungsberichte, $\mathrm{n}^{\circ} 66$, Berlin, 7-17.

Cahiers des Ingénieurs Agronomes consacré aux Landes, $\mathrm{n}^{\circ} 6,4^{*}$ tome, 1949.

DéPARTEMENT des LANDES, 1965. Inventaire forestier national, Ministère de I'Agriculture. D.G. des Eaux et Forêts. 